\title{
Article \\ A Mini-Survey and Feasibility Study of Deep-Learning-Based Human Activity Recognition from Slight Feature Signals Obtained Using Privacy-Aware Environmental Sensors
}

\author{
Hirokazu Madokoro ${ }^{1,2, *} \mathbb{C}$, Stephanie Nix ${ }^{2}$, Hanwool Woo ${ }^{3} \mathbb{C}$ and Kazuhito Sato ${ }^{2}$ \\ 1 Faculty of Software and Information Science, Iwate Prefectural University, Takizawa City 020-0693, Japan \\ 2 Faculty of Systems Science and Technology, Akita Prefectural University, Yurihonjo City 015-0055, Japan; \\ snix@akita-pu.ac.jp (S.N.); ksato@akita-pu.ac.jp (K.S.) \\ 3 Institute of Engineering Innovation, Graduate School of Engineering, The University of Tokyo, \\ Tokyo 113-8656, Japan; woo@robot.t.u-tokyo.ac.jp \\ * Correspondence: hirokazu_m@iwate-pu.ac.jp; Tel.: +81-019-694-2500
}

\section{check for}

updates

Citation: Madokoro, H.; Nix, S.; Woo, H.; Sato, K. A Mini-Survey and Feasibility Study of

Deep-Learning-Based Human

Activity Recognition from Slight

Feature Signals Obtained Using

Privacy-Aware Environmental

Sensors . Appl. Sci. 2021, 11, 11807.

https://doi.org/10.3390/

app112411807

Academic Editor: Juan Ye

Received: 15 November 2021

Accepted: 10 December 2021

Published: 12 December 2021

Publisher's Note: MDPI stays neutral with regard to jurisdictional claims in published maps and institutional affiliations.

Copyright: (c) 2021 by the authors. Licensee MDPI, Basel, Switzerland. This article is an open access article distributed under the terms and conditions of the Creative Commons Attribution (CC BY) license (https:// creativecommons.org/licenses/by/ $4.0 /)$.
Abstract: Numerous methods and applications have been proposed in human activity recognition (HAR). This paper presents a mini-survey of recent HAR studies and our originally developed benchmark datasets of two types using environmental sensors. For the first dataset, we specifically examine human pose estimation and slight motion recognition related to activities of daily living (ADL). Our proposed method employs OpenPose. It describes feature vectors without effects of objects or scene features, but with a convolutional neural network (CNN) with the VGG-16 backbone, which recognizes behavior patterns after classifying the obtained images into learning and verification subsets. The first dataset comprises time-series panoramic images obtained using a fisheye lens monocular camera with a wide field of view. We attempted to recognize five behavior patterns: eating, reading, operating a smartphone, operating a laptop computer, and sitting. Even when using panoramic images including distortions, results demonstrate the capability of recognizing properties and characteristics of slight motions and pose-based behavioral patterns. The second dataset was obtained using five environmental sensors: a thermopile sensor, a $\mathrm{CO}_{2}$ sensor, and air pressure, humidity, and temperature sensors. Our proposed sensor system obviates the need for constraint; it also preserves each subject's privacy. Using a long short-term memory (LSTM) network combined with $\mathrm{CNN}$, which is a deep-learning model dealing with time-series features, we recognized eight behavior patterns: eating, operating a laptop computer, operating a smartphone, playing a game, reading, exiting, taking a nap, and sitting. The recognition accuracy for the second dataset was lower than for the first dataset consisting of images, but we demonstrated recognition of behavior patterns from time-series of weak sensor signals. The recognition results for the first dataset, after accuracy evaluation, can be reused for automatically annotated labels applied to the second dataset. Our proposed method actualizes semi-automatic annotation, false recognized category detection, and sensor calibration. Feasibility study results show the new possibility of HAR used for ADL based on unique sensors of two types.

Keywords: activities of daily living; behavior pattern; deep learning; human activity recognition; long short-term memory

\section{Introduction \\ 1.1. Background and Scope}

Human activity recognition (HAR) is a challenging task for pattern recognition and computer vision studies, especially when using off-the-shelf camera technology [1]. Numerous applications and their derivative variations exist in the areas of multimodal gesture recognition [2], consumption and consumer behavior analysis [3], human-robot interaction [4], robotics therapy [5], and body motion analysis in sports [6]. For industrial 
applications to a security service [7] and biometric authentication [8] in a public environment, vision-based systems have been proposed for identifying suspicious people based on characteristics of facial features, facial expressions [9], and body motions [10]. Moreover, development of a vision-based system [11] and an invisible sensor system [12] for bedleaving detection and fall prevention at hospitals and nursing care sites has been reported. Such systems emphasize abnormality detection from the identification of behavior patterns.

In human living environments, real-time recognition of abnormal behavior patterns related to falls, injuries, and collisions is set as a high priority for reasons of safety and security [13]. Physical body motions in our daily life are classified roughly into three categories: standing, sitting, and lying down [14]. Standing motions are used during cooking, cleaning, household chores of various types, etc. Sitting motions are used during meals, when reading, playing games, watching television (TV), listening to music, and operating a smartphone, tablet, or laptop computer, etc. Classification and recognition of daily life behavior patterns based on image analyses is regarded as a challenging task for computer vision studies because the range of motion of represented features is limited and often inclusive of vague or meticulous movements, especially when examining sitting motions. Moreover, numerous systems use wearable devices [15] with embedded acceleration and gyro sensors without using a camera. They emphasize privacy in private environments such as living rooms.

\subsection{Sensors and Human Behavior Patterns}

For users, privacy concerns are relieved if image processing is completed inside a particularly closed device [16]. As one commercially available example, an up-to-date room air conditioner (Shirokuma-Kun series; Hitachi Ltd.; Tokyo, Japan) has a built-in wide field of view (FoV) monocular camera combined with a thermopile sensor [17]. Regarding this technical acceptance and social tendency, we consider that it will be acceptable for consumers to provide a comfortable environment using intelligent sensors. By contrast, wearable devices with physical restraints sometimes impose a mental burden on a wearer subject [18]. Moreover, the total cost for preparing, installing, and maintaining sensors installed in various places as ambient intelligence [19] is high, especially for healthcare applications [20]. For this study, we positively explore the use of cameras in daily life under the restriction that information is enclosed inside devices and systems considering mental burdens and sensor complexity. Intelligent sensor systems based on deep learning (DL) [21] provide such autonomous mechanisms and platforms.

The ultimate goal of this study is to infer an emotional state from recognized human behavior patterns in a living environment with unusual, non-routine, and extraordinary experiences. No specific definition exists for the respective patterns of usual daily living environments and unusual living environments. We assumed that unusual living environments include all locations except for those of our daily life, such as accommodations at a travel destination. Herein, university laboratories are mixed, including usual daily living environments for laboratory members and unusual living environments for non-members. Therefore, both environments are used freely depending on the observer background, situation, and context.

\subsection{Contributions}

As a preliminary study of emotion estimation applied in an unusual living environment, we developed original benchmark datasets of two types obtained in a laboratory, which is a mixed environment of usual and unusual living. For the first dataset, we used a fisheye lens camera to obtain time-series panoramic images. We specifically examine human pose estimation related to HAR as an approach that has no effect on objects because room sizes, vacant spaces, and arrangement of the furniture are generally varied and diverse in a laboratory. A particular characteristic of our method is that we employ a stateof-the-art pose estimation network to extract feature vectors from time-series panoramic images obtained using a fisheye lens camera. We also use a convolutional neural network 
(CNN) [22] to recognize five behavior patterns after classifying them as learning and verification subsets. To pursue measures to address privacy concerns, we used environmental sensors to obtain the second dataset. Experimentally obtained results demonstrate the possibility of recognizing behavior patterns of eight types from time-series tiny sensor signals using CNN combined with a short-term memory (LSTM) [23] network. Although the recognition accuracy for the second dataset was lower than that obtained for the first dataset, this paper presents an exploration of a new possibility of using HAR for activities of daily living (ADL) based on unique sensor systems and two original datasets. Accurately recognized results can be reused for automatic annotation of labels in another benchmark dataset for inferring emotion from behavior patterns. Here, the contributions of this study are as follows.

- A mini-survey presents existing HAR survey papers published between January 2016 and October 2021 and representative HAR studies reported between January 2019 and June 2021.

- Recent trends and developments of ADL studies are introduced, especially for the use of privacy-aware sensors.

- A dataset obtained using a fisheye lens monocular camera is provided with the aim reducing the burden of annotations.

- A dataset obtained using five privacy-aware environmental sensors is provided for long-term human monitoring.

The limitation of this study is that the respective dataset involves only one subject. However, we consider that this paper presents important characteristics and attributes of behavioral pattern recognition using DL-based methods.

\subsection{Outline}

This paper is structured as described below. In Section 2, we present a comprehensive survey HAR and ADL studies. For HAR, we review survey papers published between January 2016 and October 2021 and review state-of-the-art papers and proceedings published between January 2019 and June 2021. For ADL, we review details of eight representative studies. Based on investigation and analysis of these existing studies, we specifically examine tiny signal features of human motions related to HAR and ADL. Section 3 presents our first dataset, obtained using a fisheye lens camera. More than by a normal lens camera, humans are projected in small sizes. We applied two DL-based methods to five behavior patterns related to HAR. Subsequently, Section 4 presents our second dataset, obtained using air and thermometric sensors. We conducted evaluation experiments in a smaller room. We also used time-series feature changes to add three behavior patterns. Although the recognition accuracy was low compared with the first dataset, we demonstrated that these environmental sensors are useful for HAR and ADL tasks while also providing privacy and reliability. Finally, Section 5 concludes this report and highlights avenues for future work based on this feasibility study.

\section{Mini Survey}

We used Google Scholar to search for studies. All searches included the terms "human activity recognition," or "HAR" or "activities of daily living" or "ADL". First, we mainly selected open access papers. Then, we excluded articles that were less than four pages or articles with few citations published before 2021.

\subsection{Existing HAR Surveys}

For more than a quarter-century, HAR has been studied using various and numerous approaches [24]. Through that period, HAR studies have been boosted mainly by the rapid progress of computer and sensor technologies and their dissemination to our society. Numerous HAR studies have been conducted. Currently, HAR presents a challenging task because it extends to various technological domains that include, but which are not limited to, sensor selection, sensor calibration, recognition algorithm development, 
parameter optimization, real-time processing, improved accuracy, privacy consideration, and robustness for individual differences. Ultimately, HAR students examine human understanding from a behavioral perspective [25].

Numerous technical and academic papers describing HAR studies have been published each year. In addition, numerous survey papers have been presented since the advent of the first HAR studies [26-28]. Table 1 presents a summary of HAR survey papers published between January 2016 and October 2021.

We classified sensing modalities into four categories: cameras, smartphones (SM), wearable sensors (WS), and environmental sensors (ES). Although cameras are included in ES, we specifically examine them as an independent category for the following three reasons. The first reason is that they are related to an actively researched pattern recognition problem in the computer vision field [29]. The second reason is that large amounts of highdimensional pixel data can be obtained from cameras. In addition to still images, video sequences were used as spatiotemporal features for improved recognition accuracy [30]. Moreover, red, green, and blue-depth (RGB-D) cameras were widely used [31] to obtain depth data in each pixel. The third reason is the need for preservation and awareness of privacy. As a simple and convenient approach in the pursuit of privacy, image resolutions are reduced with degradation of spatial and temporal image quantity and quality. However, this measure will also degrade the resultant recognition accuracy [32].

We roughly divide recognition methods into two types: conventional machine learning (CML)-based methods and DL-based [21] methods. With respect to recognition accuracy, DL has appreciable performance and superiority over CML in numerous and varied applications [33]. The comparative application potential for real-time processing under a limited computing resource was the most important benefit of using CML. By virtue of the progress of edge computing technologies [34], cost-effective and advanced performance systems are available without using cloud computing, expensive graphics processing units (GPU), or a special analog-computing device [35].

Table 1. HAR survey papers between January 2016 and October 2021.

\begin{tabular}{lcccccccc}
\hline Pub. & Authors & \multicolumn{3}{c}{ Sensing Modality } & & \multicolumn{2}{c}{ Approach } & Num. \\
Year & (A-Z) & Camera & SM & WS & ES & CML & DL & Ref. \\
\hline 2021 & Biswal et al. [36] & $\checkmark$ & & $\checkmark$ & & $\checkmark$ & & 48 \\
2021 & Bouchabou et al. [37] & $\checkmark$ & & & $\checkmark$ & & $\checkmark$ & 113 \\
2021 & Chen et al. [38] & & $\checkmark$ & $\checkmark$ & $\checkmark$ & & $\checkmark$ & 191 \\
2021 & Mihoub et al. [39] & & & & $\checkmark$ & & $\checkmark$ & 64 \\
2021 & Muralidharan et al. [40] & $\checkmark$ & $\checkmark$ & & & $\checkmark$ & $\checkmark$ & 115 \\
2021 & Shaikh et al. [41] & $\checkmark$ & & & & & $\checkmark$ & 150 \\
2021 & Straczkiewicz et al. [42] & & $\checkmark$ & $\checkmark$ & & $\checkmark$ & $\checkmark$ & 140 \\
\hline 2020 & Beddiar et al. [43] & $\checkmark$ & & & & $\checkmark$ & $\checkmark$ & 237 \\
2020 & Carvalho et al. [44] & & $\checkmark$ & $\checkmark$ & $\checkmark$ & & & 136 \\
2020 & Dang et al. [45] & $\checkmark$ & $\checkmark$ & $\checkmark$ & $\checkmark$ & $\checkmark$ & $\checkmark$ & 236 \\
2020 & Demrozi et al. [46] & & $\checkmark$ & $\checkmark$ & & $\checkmark$ & $\checkmark$ & 219 \\
2020 & Fu et al. [47] & $\checkmark$ & & $\checkmark$ & & & & 183 \\
2020 & Hussain et al. [48] & $\checkmark$ & & $\checkmark$ & $\checkmark$ & & & 165 \\
2020 & Jung [49] & $\checkmark$ & & & & & $\checkmark$ & 60 \\
2020 & Sherafat [50] & $\checkmark$ & & $\checkmark$ & $\checkmark$ & $\checkmark$ & $\checkmark$ & 132 \\
\hline
\end{tabular}


Table 1. Cont.

\begin{tabular}{|c|c|c|c|c|c|c|c|c|}
\hline \multirow{2}{*}{$\begin{array}{l}\text { Pub. } \\
\text { Year }\end{array}$} & \multirow{2}{*}{$\begin{array}{c}\text { Authors } \\
\text { (A-Z) }\end{array}$} & \multicolumn{4}{|c|}{ Sensing Modality } & \multicolumn{2}{|c|}{ Approach } & \multirow{2}{*}{$\begin{array}{l}\text { Num. } \\
\text { Ref. }\end{array}$} \\
\hline & & Camera & SM & WS & ES & CML & DL & \\
\hline 2019 & Dang et al. [51] & & $\checkmark$ & $\checkmark$ & & & & 187 \\
\hline 2019 & Dhiman et al. [52] & $\checkmark$ & & & & $\checkmark$ & $\checkmark$ & 208 \\
\hline 2019 & Elbasiony et al. [53] & & $\checkmark$ & & & $\checkmark$ & & 48 \\
\hline 2019 & Hussain et al. [54] & & & & $\checkmark$ & & & 141 \\
\hline 2019 & Jobanputra et al. [55] & & & & & $\checkmark$ & & 9 \\
\hline 2019 & Li et al. [56] & & & & $\checkmark$ & & $\checkmark$ & 106 \\
\hline 2019 & Lima et al. [57] & & $\checkmark$ & $\checkmark$ & & & $\checkmark$ & 149 \\
\hline 2019 & Slim et al. [58] & & $\checkmark$ & $\checkmark$ & & $\checkmark$ & $\checkmark$ & 119 \\
\hline 2019 & Wang et al. [59] & & & $\checkmark$ & $\checkmark$ & & $\checkmark$ & 77 \\
\hline 2018 & Nweke et al. [60] & & & $\checkmark$ & & & $\checkmark$ & 275 \\
\hline 2018 & Ramamurthy et al. [61] & & $\checkmark$ & $\checkmark$ & & $\checkmark$ & $\checkmark$ & 83 \\
\hline 2018 & Shickel et al. [62] & & & $\checkmark$ & & $\checkmark$ & $\checkmark$ & 63 \\
\hline 2018 & Wang et al. [63] & $\checkmark$ & & & & & $\checkmark$ & 182 \\
\hline 2017 & Cornacchia et al. [64] & & $\checkmark$ & $\checkmark$ & & $\checkmark$ & & 225 \\
\hline 2017 & Chen et al. [65] & $\checkmark$ & & $\checkmark$ & & & & 78 \\
\hline 2017 & Morales et al. [66] & & $\checkmark$ & $\checkmark$ & & & & 65 \\
\hline 2017 & Rault et al. [67] & & $\checkmark$ & $\checkmark$ & & & & 88 \\
\hline 2017 & Vyas et al. [68] & & $\checkmark$ & & & & & 39 \\
\hline 2016 & Dawn et al. [69] & $\checkmark$ & & & & $\checkmark$ & & 66 \\
\hline 2016 & Onofri et al. [70] & $\checkmark$ & & & & & & 90 \\
\hline
\end{tabular}

\subsection{State-of-the-Art HAR Studies}

Although several survey papers $[43,45,46,52,60,64]$ have cited more than 200 related research papers, these represent just a fraction of the numerous HAR studies that have been conducted to date. The numerous papers show the limited terms of years and fields of applications, including overlapping scopes among the survey papers. Moreover, several survey papers have reported earlier reports that present a comprehensive literature review. For this research field, numerous research and survey papers have been published over a short period. That trend is expected to continue. For our mini-survey, Table 2 presents representative HAR studies reported between January 2019 to June 2021. We specifically emphasized a review of recognition methods in the third column and benchmark datasets in the fourth column.

Table 2. Representative HAR studies reported between January 2019 and June 2021.

\begin{tabular}{cccc}
\hline Year & Authors & Methods & Datasets \\
\hline 2021 & Fu et al. [71] & IPL-JPDA & original \\
2021 & Gorji et al. [72] & CML (RF) & original \\
2021 & Gul et al. [73] & YOLO+CNN & original \\
2021 & Hussain et al. [74] & CML (RF) & {$[123]$} \\
2021 & Mekruksavanich et al. [75] & 4-CNN-LSTM & {$[124]$} \\
2021 & Mekruksavanich et al. [76] & CNN-LSTM & {$[124,125]$} \\
2021 & Moreira et al. [77] & ConvLSTM & original \\
2021 & Nafea et al. [78] & CNN-BiLSTM & {$[124,126]$} \\
2021 & Xiao et al. [79] & DIM-BLS & {$[124,126]$} \\
\hline 2020 & Ahmed et al. [80] & CML (SVM) & {$[127]$} \\
2020 & Ashry et al. [81] & BLSTM & original \\
2020 & Debache et al. [82] & CML and CNN & original \\
2020 & Ehatisham-Ul-Haq et al. [83] & CML & {$[128]$} \\
\hline
\end{tabular}


Table 2. Cont.

\begin{tabular}{|c|c|c|c|}
\hline Year & Authors & Methods & Datasets \\
\hline 2020 & Ferrari et al. [84] & Adaboost+CNN & [129-131] \\
\hline 2020 & Hamad et al. [85] & CNN-LSTM & [132-134] \\
\hline 2020 & Ihianle et al. [86] & MCBLSTM & {$[135,136]$} \\
\hline 2020 & Irvine et al. [87] & EL & [123] \\
\hline 2020 & Khannouz et al. [88] & CML & {$[135,137]$} \\
\hline 2020 & Lawal et al. [89] & $\mathrm{CNN}$ & [138] \\
\hline 2020 & Machot et al. [90] & ZSL & [139] \\
\hline 2020 & Mukherjee et al. [91] & CNN (ResNet-101) & [140]+original \\
\hline 2020 & Mutegeki et al. [92] & CNN-LSTM & [124] \\
\hline 2020 & Pham et al. [93] & CNN-LSTM & [141-143] \\
\hline 2020 & Popescu et al. [94] & $\mathrm{CNN}$ & [141-143] \\
\hline 2020 & Qin et al. [95] & CNN (Fusion-ResNet) & {$[135,144]$} \\
\hline 2020 & Shrestha et al. [96] & BLSTM & original \\
\hline 2020 & Taylor et al. [97] & CML & original \\
\hline 2020 & Tanberk et al. [98] & 3D-CNN-LSTM & original \\
\hline 2020 & Wan et al. [99] & $\mathrm{CNN}$ & [124] \\
\hline 2020 & Wang et al. [100] & H-LSTM & [124] \\
\hline 2020 & Xia et al. [101] & LSTM-CNN & {$[126,145,146]$} \\
\hline 2020 & Xu et al. [102] & CNN (Fusion-Mdk-ResNet) & {$[124,126,147]$} \\
\hline 2019 & Chung et al. [103] & LSTM & original \\
\hline 2019 & Concone et al. [104] & HMM & original \\
\hline 2019 & Ding et al. [105] & CNN+TL & {$[125,148]$} \\
\hline 2019 & Ding et al. [106] & RNN (LSTM) & original \\
\hline 2019 & Gumaei et al. [107] & RNN & [149] \\
\hline 2019 & Ehatisham-Ul-Haq et al. [108] & CML ( $k$ NN, SVM) & [142] \\
\hline 2019 & Gani et al. [109] & GMM & original \\
\hline 2019 & Li et al. [110] & BLSTM & original \\
\hline 2019 & Kim et al. [111] & HMM & original \\
\hline 2019 & Naveed et al. [112] & CML (SVM) & {$[150,151]$} \\
\hline 2019 & Qi et al. [113] & CNN & original \\
\hline 2019 & Siirtola et al. [114] & EL & original \\
\hline 2019 & Tian et al. [115] & CML (SVM+ELM) & original \\
\hline 2019 & Voicu et al. [116] & CML (SVM) & original \\
\hline 2019 & Xu et al. [117] & EL & [124] \\
\hline 2019 & Xu et al. [118] & EL & [147] \\
\hline 2019 & Yang et al. [119] & CNN (Inception) & {$[124,147,152]$} \\
\hline 2019 & Zebin et al. [120] & CNN (LeNet) & original \\
\hline 2019 & Zhang et al. [121] & U-Net & {$[126,145,146]+$ original } \\
\hline 2019 & Zhu et al. [122] & EL & original \\
\hline
\end{tabular}

The most commonly used method is CNN-LSTM (e.g., 9 out of 51 studies): a model based on CNN with various backbones combined partially with LSTM [23] layers, including ConvLSTM [153]. Bidirectional LSTM (BLSTM) [154] was used solely or combined with CNN as CNN-BLSTM. Furthermore, CML-based and DL-based ensemble learning (EL), which comprise bagging, boosting, and stacking, are used commonly as HAR methods. For CML-based methods, support vector machines (SVM) [155], random forests (RF) [156], and $k$ nearest neighbors $(k N N)$ were used. Various $\mathrm{CNN}$ backbones have been used: LeNet [157], Inception [158], VGG-Net [159], Xception [160], ResNet [161], and ResNet's derivative models in parentheses if they are indicated explicitly in the references. For other methods, various models and algorithms have been used: improved pseudo-labels joint probability domain adaptation (IPL-JPDA), you look only once (YOLO) [162], multichannel bidirectional LSTM (MCBLSTM), extreme learning machine (ELM) [163], zero-shot learning (ZSL) [164], and U-Net [165].

Open datasets for HAR studies and competitions are well maintained for benchmarking $[123-152,166,167]$. Advantages of using open benchmark datasets are not only 
that it saves time and costs for the development of sensor systems and data collection; it also facilitates performance comparison with other state-of-the-art methods. By contrast, because accuracy improvements have become saturated, the performance competition is becoming increasingly severe year by year. We regard original dataset developments for various scenarios, environments, and target persons as a fundamental and important research element for the wide varieties and scopes of HAR practical applications. Especially in recent years, the performance, duration, and size reduction in sensors are improving constantly. In addition to using commercially available sensors, the use of originally developed handmade sensors [12] can be expected to yield new applications according to applicable environments and restrictions. Although original benchmark datasets require a huge workload for data collection and their labeling for annotation, the development of an automatic data collection platform using an internet of things (IoT) [168] sensor devices provides a solution for workload reduction. Extension of studies and their applications can be anticipated in the furtherance of healthcare management [169], privacy consideration [170], and multimodal sensing [171] as advanced original system development [172].

\subsection{Recent Trends and Developments of ADL Studies}

Various methods for estimating dominant emotions [173] have been reported from various motions, actions, and behavior patterns. Nguyen et al. [174] widely surveyed video-based human behavior recognition studies related to ADL [175]. The results have been useful in supporting ambient assisted living systems for elderly people living independently. Generally, ADL patterns have included combing hair, applying makeup, brushing teeth, dental flossing, washing hands or face, drying hands or face, doing laundry, washing dishes, moving dishes, making tea, making coffee, drinking water or from a bottle, drinking water or from a tap, making a cold food snack, vacumming a floor, watching TV, using a computer, and using a cellular telephone [176]. To support ADL, they analyzed technical difficulties, such as occlusions and limited FoV, of conventional camera systems used in public environments. Moreover, they described that object recognition remained as a task for future study because intra-class variation occurred in unconstrained ADL scenarios. Therefore, they concluded that current system performance is far from adequate.

To enhance privacy and data capacity, accelerometers and gyro sensors can be used instead of cameras. Chelli et al. [177] developed an ML-based framework that is useful for fall detection and ADL recognition. For their study, ADL was set as comprising six categories: standing, sitting, walking, walking upstairs, walking downstairs, and lying down. They compared four popular CML algorithms: a multilayer perceptron (MLP), $k \mathrm{NN}$, quadratic SVM, and ensemble bagged trees (EBT). The experimentally obtained results using a dataset produced for use with a smartphone revealed that the accuracy of fall detection reached $100 \%$ for both quadratic SVM and EBT algorithms.

Based on behavioral biometrics, Weiss et al. [136] proposed a recognition method for 18 physical activities including walking, jogging, using stairs, sitting, standing, kicking a soccer ball, dribbling a basketball, catching a tennis ball, typing, writing, clapping, brushing teeth, folding clothes, eating pasta, eating soup, eating a sandwich, eating chips, and drinking from a cup. The experimentally obtained results obtained with their original benchmark dataset using accelerometers and gyroscope sensors on a smartphone and a smartwatch revealed that zero-effort continuous biometrics based on normal activities and certain easy-to-perform activities of daily living are feasible and viable for gait-based biometrics. Nevertheless, their method involved wide-body motions, especially for hands and legs. Therefore, as a shortcoming, accuracy was markedly low for small and restricted motions done in a sitting posture such as eating, reading, and studying.

One important application of human motion recognition technology is for healthsmart homes, which have various heterogeneous sensors. Diraco et al. [178] proposed a DL-based method for detecting early changes in human behaviors. The method specifically examines starting, duration, disappearing, swapping, locating, and heart rate changes. For actualizing long-term continuous monitoring, they used multiple sensors such as stereo 
cameras, monocular cameras, time of flight (TOF) cameras, microphones, pyroelectric infrared (PIR), sonar, light detection and ranging (LiDAR), ultra-wideband LiDAR, strain gauges, barometers, and vibration sensors. The experimentally obtained results demonstrated superiority in terms of accuracy and calculation time for detection and prediction. Unsupervised DL-based deep clustering (DC) methods were found to be superior to traditional supervised and semi-supervised methods using SVM including one-class SVM, $\mathrm{CNN}$, stacked auto-encoders (SAE), and $k$-means clustering. Nevertheless, the burdens related to annotation for long-term continuous monitoring datasets remain difficult for practical application and dissemination.

To associate emotions and behavior patterns recognized from images, benchmark datasets were provided: interactive emotional dyadic motion capture (IEMO-CAP) datasets [179], the multimodal corpus of sentiment intensity and subjectivity analysis in online opinion videos (MOSI) [180], one-minute-gradual (OME) emotion datasets, emotion recognition in the wild named EmotiW [181], and the affect-net database [182]. For these datasets, the associated emotions comprise disgust, fear, happiness, surprise, sadness, and anger defined by Ekman [183]. Combined with these six basic emotion categories, Nojavanasghari et al. [184] added nine complex emotion categories: curiosity, uncertainty, excitement, attentiveness, exploration, confusion, anxiety, embarrassment, and frustration. Moreover, Weixuan et al. [185] added 11 complex emotional categories: amusement, contempt, contentment, embarrassment, excitement, guilt, pleasure, pride, relief, satisfaction, and shame.

As a smart classroom application at an elementary school, Kim et al. [186] proposed a system that includes affective sensing, DL-based emotion recognition, and real-time mobilecloud computing networks. Their system yielded real-time suggestions with an in-class presenter to improve the quality and memorability of their allowed presentation. Moreover, their system yielded real-time adjustments and corrections to their non-verbal behavior, such as hand gestures, facial expressions, and body language. Their comprehensive study found computational requirements for their proposed system, which incorporates these technologies. Based on these requirements, they assess current difficulties and suggest some future directions in engineering and education disciplines for system deployment. They merely designed a conceptual system and its framework without specific classification or recognition of motion or behavior patterns.

Using a depth camera for three-dimensional (3D) human pose reconstruction, Marinoiu et al. [187] introduced a challenging task to recognize emotions from fine-grained actions. They developed non-staged videos recorded during robot-assisted therapy sessions for children with autism, along with non-standard camera viewpoints. Based on this development, they presented challenges: a large dataset with long videos, numerous highly variable actions, children who are only partially visible, and children of different ages who might show unpredictable actions. They investigated 3D human pose reconstruction methods for newly introduced tasks. Furthermore, they proposed extensions to adapt them to address these challenges. Moreover, for establishing several baselines and implications in the context of a child-robot interaction, they analyzed multiple approaches to action and emotion recognition from 3D human pose features.

For extraction of emotion-specific features from a vast number of human body motion descriptors, Ahmed et al. [188] introduced a double layer feature selection framework to classify emotions from a comprehensive list that included body motion features of 10 types. They used that feature selection framework to achieve accurate recognition of five basic emotions of happiness, sadness, fear, anger, and neutral, combined with three scenarios of walking, sitting, and action-independent scenarios. They validated their method using open datasets [189] obtained using a depth camera from 30 subjects. The experimentally obtained results revealed that their proposed emotion recognition system achieved excellent emotion recognition. In fact, it outperformed five widely used CML methods: SVM, linear discriminant analysis (LDA), naive Bayes (NB) classifier, $k N N$, and 
decision tree (DT). Nevertheless, its recognition accuracy was strongly dependent on the scenarios which were set in advance.

\section{Vision-Based Approach Using a Fisheye Lens Monocular Camera}

\subsection{Proposed Sensor System and Recognition Method}

Figure 1 depicts procedures used for our first proposed method. The procedures are divided roughly into three steps. Time-series panoramic images are obtained using a fisheye lens camera in the first step. For this measurement, we fixed the camera at a position $x \mathrm{~m}$ distant from a subject. The obtained images were downsampled to $n \mathrm{fps}$ with a linear transformation to reduce the computation time and memory size.

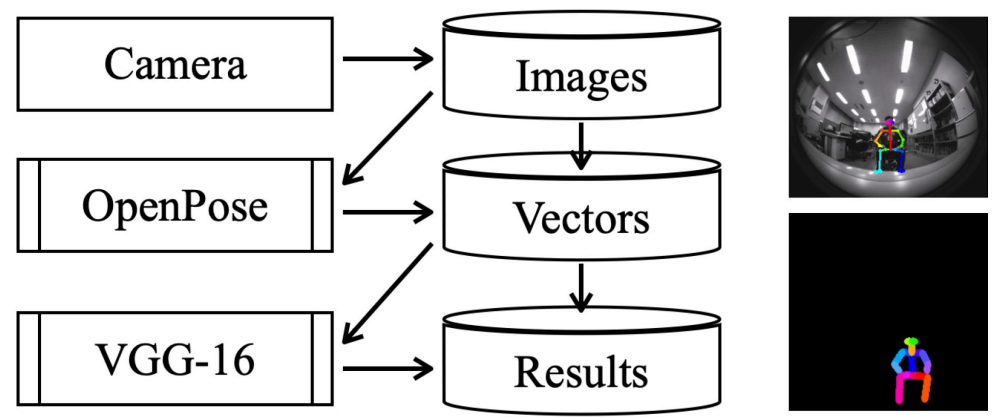

Figure 1. Comprehensive system architecture of the proposed procedure.

The second step uses OpenPose [190] to extract feature vectors of pose motions. Herein, human two-dimensional (2D) pose estimation represents a challenging task for computer vision studies. Numerous methods [191-199] have been proposed to detect 2D poses from multiple people in a single image. Based on an earlier study reported by Noori et al. [200], we used OpenPose [190] because of its simple and easy implementation, high-speed processing, and high estimation accuracy. Regarding the accuracy verification of OpenPose, Kim et al. [201] employed 17 inertial sensors to evaluate 12 motion tasks: upright standing, trunk flexion $30^{\circ}$, sitting on a stool trunk flexed and rotated, placing an object on top, kneeling above the head, arms crossed, holding a box, legs crossed, sitting at the desk, simple lifting, and complex lifting.

OpenPose [190] employs a bottom-up representation of association scores via part affinity fields (PAFs) [202]. A set of 2D vector fields is encoded for the location and orientation of limbs over an image domain. To detect body parts, confidence maps are predicted from two-branch CNN. As an open library, OpenPose was trained using two benchmark datasets for multi-person pose estimation: The Max Planck Institut Informatik (MPII) human pose dataset [203] and the common objects in context (COCO) key-points challenge dataset [204]. For our implementation, we used the OpenPose library, which was pre-trained using the latter dataset.

As examined for behavior pattern classification and recognition used for labeling, we used the VGG-16 [159] backbone, which comprises 13 convolutional layers and three fully connected layers. The original VGG-16 was designed as a deep architecture to use $3 \times 3$ convolution filters for each pixel. Using feature vectors produced from OpenPose, we applied transfer learning [205] to the upper layers of VGG-16, which were trained in advance using ImageNet [206]. Although ResNet [161] and its derivative models such as WideResNet [207], ResNeXt [208], Res2Net [209], and Inception-resnet (Inception-v4) [210] have already been proposed, we used the VGG-Net backbone because of our overall considerations of GPU time, speed, and memory consumption [211]. Actually, CNN backbones can be switched easily according to specifications and applications. 
Table 3 presents the major parameters of VGG-16. Based on our earlier study [212], the number of generations, batch size, and validation split were set, respectively, to 50 epochs, 4 , and 0.2 . The number of the input layer corresponds to the resized image size: 224 pixels $\times 224$ pixels $\times 3$ channels. The numbers of the output layer were set to two, three, and five, according to the number of target categories. For the optimization algorithm, we used Adam [213], which is an optimizer combined with Momentum [214] and RMSProp [215].

Table 3. VGG-16 parameters and their setting values.

\begin{tabular}{cc}
\hline Parameters & Setting Values \\
\hline Learning iteration & 50 epochs \\
Batch size & 4 \\
Validation split & 0.2 \\
Num. input layer & $224 \times 224 \times 3$ units \\
Num. output layer & 2,3, or 5 units \\
Optimization algorithms & Adam [213] \\
\hline
\end{tabular}

\subsection{Setup and the Obtained First Dataset}

Table 4 presents some major specifications of the fisheye lens camera (RealSense T265; Intel Corp.; Santa Clara, CA, USA) used for this study. The FoV of this camera is $63 \pm 5 \mathrm{deg}$, which is approximately twice that of an ordinary monocular camera. The camera body is $12.5 \mathrm{~mm}$ long $\times 108 \mathrm{~mm}$ wide $\times 24.5 \mathrm{~mm}$ high. This compact camera can reduce the psychological burdens of a subject that might result from awareness of a camera during an experiment. Nevertheless, because of this wide FoV, the subject becomes extremely small if the camera is in a distant position. In addition, image distortion is extensive at the image edges. For this property, we set $x=2.0$ in specific consideration of this camera.

Table 4. Major specifications of the fisheye lens camera (RealSense T265; Intel Corp.).

\begin{tabular}{cc}
\hline Parameters & Specifications \\
\hline Imaging sensor & OV9282 \\
Lens size & 14 inch \\
Pixel size & $3 \times 3 \mu \mathrm{m}^{2}$ \\
FoV & $163 \pm 5 \mathrm{deg}$ \\
Resolution & $\mathrm{W} 1280 \times \mathrm{H} 800$ pixels \\
Frame rate & $120 \mathrm{fps}$ \\
IMU & BMI055 \\
Body dimensions & $512.5 \times \mathrm{W} 108.0 \times \mathrm{H} 24.5 \mathrm{~mm}$ \\
Weight & $1.5 \mathrm{~W}$ \\
\hline
\end{tabular}

We obtained images for the dataset in our university laboratory. The $100 \mathrm{~m}^{2}$ room, which has 17 students and 2 researchers, is an open space with no blocking structure except for $1.2 \mathrm{~m}$ high partitions. In a meeting corner of this room, we set up an environment for obtaining images.

The ultimate goal of this line of research is to infer a person's emotional state based on their displayed behavior patterns. Human emotions have individual and often idiosyncratic differences that present implications about the person's affect, well-being, and social relations [216]. For this study, we aim at developing an individual estimation model that is suitable and specialized for each subject without using a common and general estimation model. 
The target behavior patterns represent five categories: eating (ET), reading (RD), operating a smartphone (SP), operating a laptop computer (LC), and sitting (ST). Herein, ST stands for a state of doing nothing while sitting. Moreover, ST might represent simple relaxation, taking a break, taking a nap, thinking, or dreaming. Recognition of human activities that involve great amounts of motion during standing has already been addressed in a report described by Noori et al. [200]. Therefore, for this study, we specifically examined slight motions that are made during sitting. After we explained the ethics and purpose of this experiment, the subject performed these five actions in order. The interval of each behavior was set as approximately $20 \mathrm{~s}$.

Table 5 presents details of the obtained images as our original dataset, which contains 10 subsets. Each subset was obtained twice for each behavior pattern. The behavior periods varied slightly because of the lack of a timer setting. Although all images included the subject, several unconvertible images of feature vectors occurred in LC and ST. All feature vectors were extracted from three categories: ET, RD, and SP. The conversion ratio for all datasets was 0.92 for this dataset.

Table 5. Details of our original benchmark dataset.

\begin{tabular}{|c|c|c|c|c|c|}
\hline $\begin{array}{c}\text { Behavior } \\
\text { Pattern }\end{array}$ & $\begin{array}{l}\text { Subset } \\
\text { Name }\end{array}$ & $\begin{array}{l}\text { Capture } \\
\text { Time (s) }\end{array}$ & $\begin{array}{l}\text { Total Images } \\
\text { (Frames) }\end{array}$ & $\begin{array}{l}\text { Valid Images } \\
\text { (Frames) }\end{array}$ & $\begin{array}{c}\text { Conversion } \\
\text { Ratio }\end{array}$ \\
\hline \multirow[t]{2}{*}{$\mathrm{ET}$} & ET1 & 21 & 218 & 218 & 1.00 \\
\hline & ET2 & 20 & 209 & 209 & 1.00 \\
\hline \multirow[t]{2}{*}{ RD } & RD1 & 20 & 200 & 200 & 1.00 \\
\hline & RD2 & 20 & 200 & 200 & 1.00 \\
\hline \multirow[t]{2}{*}{ SP } & SP1 & 20 & 202 & 202 & 1.00 \\
\hline & SP2 & 20 & 202 & 202 & 1.00 \\
\hline \multirow[t]{2}{*}{ LC } & LC1 & 20 & 202 & 202 & 1.00 \\
\hline & LC2 & 20 & 204 & 172 & 0.84 \\
\hline \multirow[t]{2}{*}{ ST } & ST1 & 18 & 181 & 138 & 0.76 \\
\hline & ST2 & 16 & 160 & 100 & 0.63 \\
\hline \multicolumn{2}{|c|}{ Total } & 199 & 1978 & 1843 & 0.93 \\
\hline
\end{tabular}

Table 6 presents major performance specifications of our computer, which was used to execute OpenPose [190] and VGG-16 [159]. For this study, we set $n=10$ with emphasis on our computer environment. Table 7 presents details of the computational time necessary to calculate feature vectors using OpenPose. The total computational time for 2214 images was $182,241 \mathrm{~s}$. The average computational time per frame was $85.11 \mathrm{~s}$. The computational times differed depending on the respective action patterns. The shortest and longest were, respectively, $57.98 \mathrm{~s}$ in SP1 and $135.99 \mathrm{~s}$ in ST2.

Table 6. Major specifications of the computer used for this experiment.

\begin{tabular}{cc}
\hline Items & Specifications \\
\hline OS & Windows 10 Professional 64 bit; Microsoft Corp. \\
CPU & Core i5-6200 (2.30 GHz); Intel Corp. \\
Memory & $8192 \mathrm{MB}$ \\
GPU & HD Graphics 520; Intel Corp. \\
VRAM & $128 \mathrm{MB}$ \\
\hline
\end{tabular}


Table 7. Details of processing used to extract feature vectors using OpenPose.

\begin{tabular}{|c|c|c|c|c|}
\hline $\begin{array}{c}\text { Behavior } \\
\text { Pattern }\end{array}$ & $\begin{array}{l}\text { Subset } \\
\text { Name }\end{array}$ & $\begin{array}{c}\text { Processing } \\
\text { Time (s) }\end{array}$ & $\begin{array}{l}\text { Total Images } \\
\text { (Frame) }\end{array}$ & $\begin{array}{l}\text { Second per } \\
\text { Frame (s) }\end{array}$ \\
\hline \multirow[t]{2}{*}{ ET } & ET1 & 20,949 & 218 & 96.10 \\
\hline & ET2 & 21,659 & 209 & 103.63 \\
\hline \multirow[t]{2}{*}{ RD } & RD1 & 22,992 & 200 & 114.96 \\
\hline & RD2 & 12,056 & 200 & 60.28 \\
\hline \multirow[t]{2}{*}{ SP } & SP1 & 11,711 & 202 & 57.98 \\
\hline & SP2 & 11,757 & 202 & 58.20 \\
\hline \multirow[t]{2}{*}{ LC } & LC1 & 11,982 & 202 & 59.32 \\
\hline & LC2 & 11,750 & 172 & 68.31 \\
\hline \multirow[t]{2}{*}{ ST } & ST1 & 18,409 & 138 & 133.40 \\
\hline & ST2 & 13,599 & 100 & 135.99 \\
\hline \multicolumn{2}{|c|}{ Total } & 156,864 & 1843 & 85.11 \\
\hline
\end{tabular}

Figure 2 depicts sample images of ET, RD, SP, LC, and ST. In each image, the left, center, and right panels, respectively, depict the original, pose detection, and feature vector sub-images. Except for ST, occlusion occurred because of a desk. Although the subject's legs were not included, the skeleton position of the upper buttocks was estimated. Although the laptop display occluded the arms, the second joints were estimated. Images show that differences between the ET and SP postures are slight. The ET and SP images depict that differences between the postures are slight.
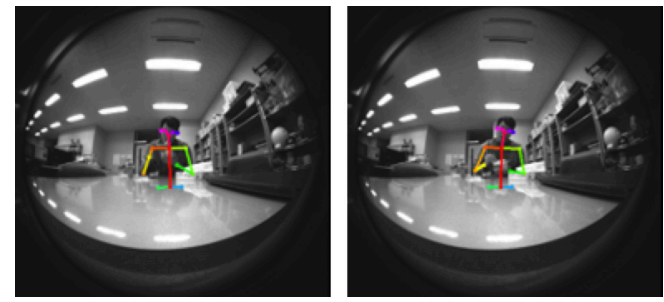

(a) ET
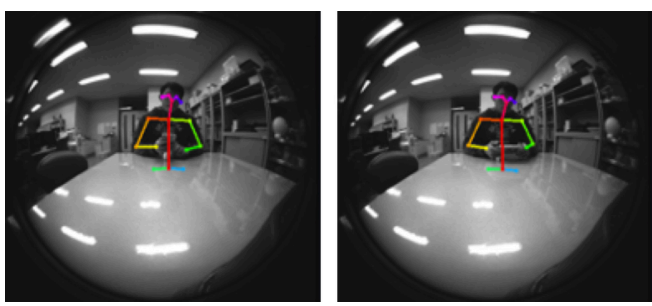

(c) $\mathrm{SP}$

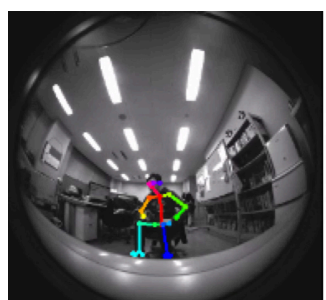

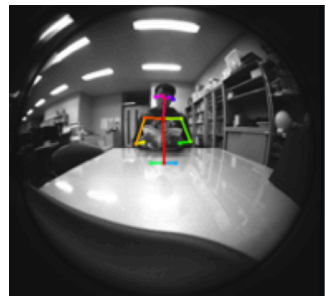

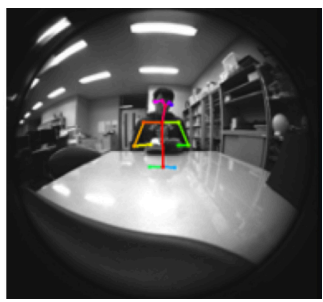

(b) RD
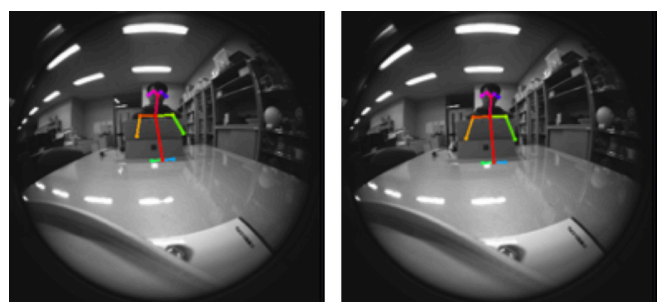

(d) LC

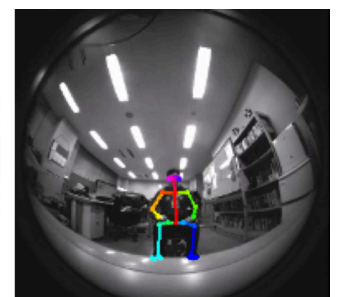

(e) ST

Figure 2. Sample images of eating (ET), reading (RD), operating a smartphone (SP), operating a laptop computer (LC), and sitting (ST). 


\subsection{Experiment Results and Discussion}

Using our original datasets combined with five behavior patterns, we conducted three experiments to evaluate the recognition accuracy and loss. Herein, $A$ and $L$, respectively, denote accuracy and loss. Moreover, we set $A_{t r a}, A_{\text {val }}, L_{\text {tra }}$, and $L_{v a l}$ as, respectively, representing accuracy for training datasets, accuracy for validation datasets, loss for training dataset, and loss for validation datasets. Table 8 presents the combinations of behavior patterns for experiments among the designated Experiments A, B, and C. As the minimum combination, Experiment A assesses two behavior patterns: ET and RD. Experiment $B$ represents a combination of Experiment $A$ and SP. Experiment $C$ targets all five behavior patterns. The first-round subsets, which comprise ET1, RD1, SP1, LC1, and ST1, are used for training. The second-round subsets, which comprise ET2, RD2, SP2, LC2, and ST2, are used for training. The number of training iterations was set as 10 epochs for all experiments.

Table 8. Combinations of behavior patterns for the respective experiments.

\begin{tabular}{cccccc}
\hline Experiment & ET & RD & SP & LC & ST \\
\hline A & $\checkmark$ & $\checkmark$ & & & \\
B & $\checkmark$ & $\checkmark$ & $\checkmark$ & & \\
C & $\checkmark$ & $\checkmark$ & $\checkmark$ & $\checkmark$ & $\checkmark$ \\
\hline
\end{tabular}

The evaluation dataset used for Experiment A comprises ET and RD of two behavior patterns. Figure 3 presents transitions of $A_{\text {tra }}, A_{\text {val }}, L_{t r a}$, and $L_{\text {val }}$ in 10 epochs. Overall, the data show that $A_{\text {tra }}$ increased steadily. The value of $A_{\text {val }}$ soared during the fourth epoch. Although a momentary drop occurred in the fifth epoch, $A_{\text {val }}$ increased steadily. Eventually, both $A_{\text {tra }}$ and $A_{\text {val }}$ reached 1.000 at the seventh epoch. By contrast, $L_{\text {tra }}$ and $L_{v a l}$ decreased steadily.

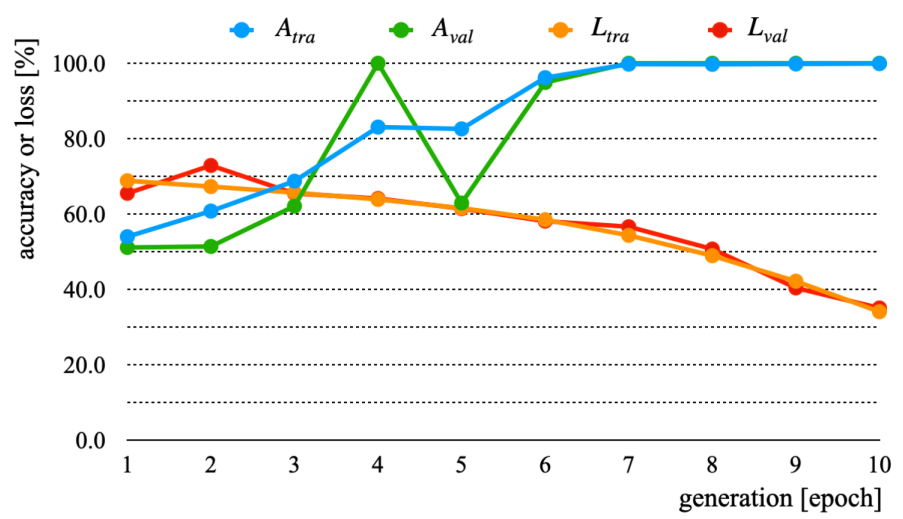

Figure 3. Transitions of $A_{t r a}, A_{v a l}, L_{t r a}$, and $L_{v a l}$ from Experiment A.

Evaluation datasets on Experiment B comprise ET, RD, and SP. This combination is equivalent to the addition of SP to Experiment A. Although the subject grasped different objects in RD and SP, the feature vectors obtained using OpenPose [190] were very similar. For that reason, we specifically examined the recognition and verification of RD and SP.

Figure 4 presents transitions of $A_{\text {tra }}, A_{v a l}, L_{t r a}$, and $L_{v a l}$ in 10 epochs. Both $A_{\text {tra }}$ and $A_{\text {val }}$ increased steadily. The values of $L_{\text {tra }}$ and $L_{\text {val }}$ decreased, respectively, from the sixth epoch and the fourth epoch. After increasing $L_{v a l}$ in the fifth epoch, it decreased from the sixth epoch. 


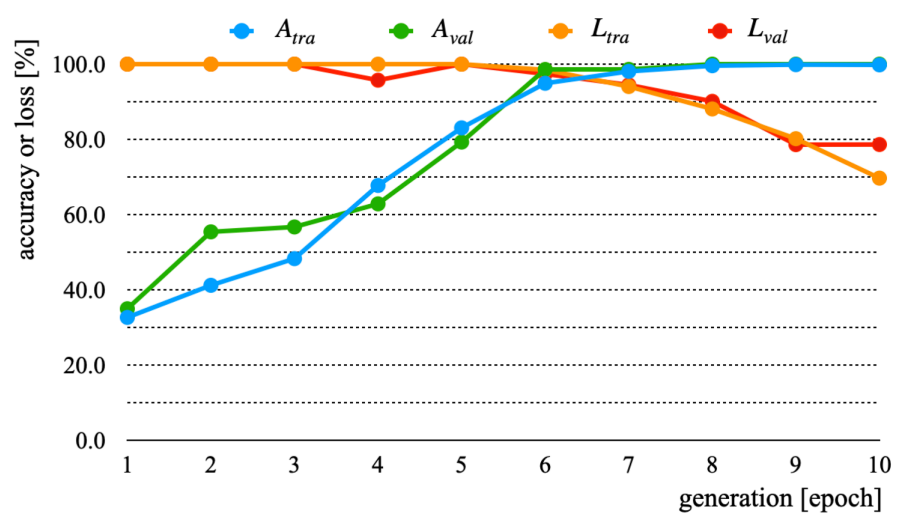

Figure 4. Transitions of $A_{t r a}, A_{v a l}, L_{t r a}$, and $L_{v a l}$ for Experiment B.

We conducted Experiment $\mathrm{C}$ using all images in the five categories. Figure 5 presents transitions of $A_{\text {tra }}, A_{\text {val }}, L_{\text {tra }}$, and $L_{\text {val }}$ in 10 epochs. The value of $A_{\text {tra }}$ increased steadily. The value of $A_{\text {val }}$ increased steadily, except for a slight drop in the fourth epoch. Both $L_{t r a}$ and $L_{v a l}$ decreased steadily from the seventh epoch.

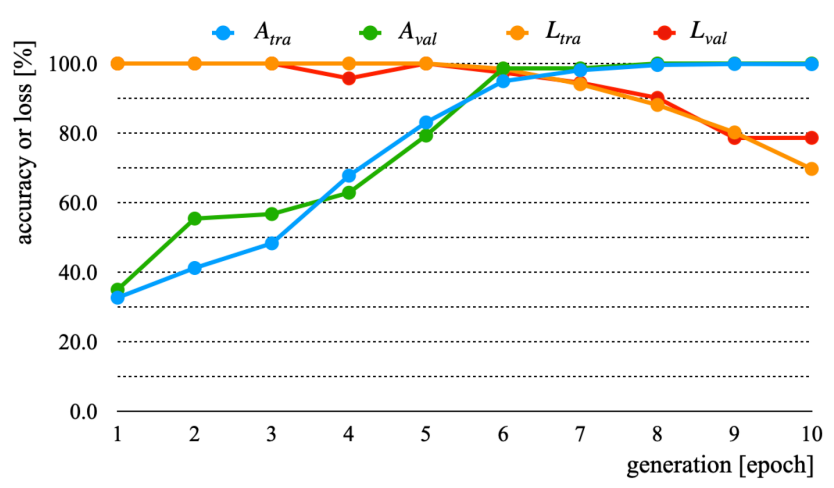

Figure 5. Transitions of $A_{\text {tra }}, A_{\text {val }}, L_{t r a}$, and $L_{v a l}$ for Experiment C.

Table 9 presents the confusion matrix obtained from Experiment C. One image of RD was falsely recognized as ET. One image of LC was falsely recognized as SP. All of the other 881 images were recognized correctly.

Table 9. Confusion matrix from Experiment C (frames).

\begin{tabular}{cccccc}
\hline Category & ET & RD & SP & LC & ST \\
\hline ET & 209 & 0 & 0 & 0 & 0 \\
RD & 1 & 199 & 0 & 0 & 0 \\
SP & 0 & 0 & 202 & 0 & 0 \\
LC & 0 & 0 & 1 & 171 & 0 \\
ST & 0 & 0 & 0 & 0 & 100 \\
\hline
\end{tabular}

Table 10 presents a summary of all results obtained for $A_{\text {tra }}, A_{\text {val }}, L_{\text {tra }}$, and $L_{\text {val }}$ from the respective experiments. Regarding $A$ for all experiments, both $A_{\text {tra }}$ and $A_{\text {val }}$ were saturated around the seventh epoch. The $L$ curve shows that this parameter tends to decrease if the number of iterations is increased by more than 10 epochs. Nevertheless, because of the decreased number of $L$, overfitting might occur if the number of iterations increases for training with sufficient accuracy of $A$. For this study, we obtained subsets of the dataset twice in a short time for one subject. Obtaining a new dataset with different dates and times is important to evaluate the overlearning and generalization that occur with our method. 
Table 10. Results of accuracy and loss obtained from the respective experiments (\%).

\begin{tabular}{ccccc}
\hline Experiment & $\boldsymbol{A}_{\text {tra }}$ & $\boldsymbol{A}_{\text {val }}$ & $\boldsymbol{L}_{\text {tra }}$ & $\boldsymbol{L}_{\text {val }}$ \\
\hline A & 100.0 & 100.0 & 34.1 & 35.1 \\
$\mathrm{~B}$ & 100.0 & 100.0 & 69.7 & 78.6 \\
C & 99.3 & 99.7 & 55.0 & 53.9 \\
\hline
\end{tabular}

\section{Environmental Approach Using Air and Thermometric Sensors}

\subsection{Proposed Sensor System and Recognition Method}

For the second approach, we aimed at developing a privacy-aware sensor system. Figure 6a depicts the overall configuration of our second proposed system. We selected five environmental sensors that measure air and temperature distributions. A single-board computer (SBC), Raspberry Pi 4 Model B, was used to save measurement signals to a flash memory device. This SBC is a commonly and widely used prototyping device available for scientific research and industrial application development [217]. Measurement signals obtained from two independent sensor modules are sent to an IoT cloud service called Ambient (AmbientData Inc.; Tokyo, Japan) for storage and visualization. The data-receiving interval of this free service is $1 \mathrm{~min}$.

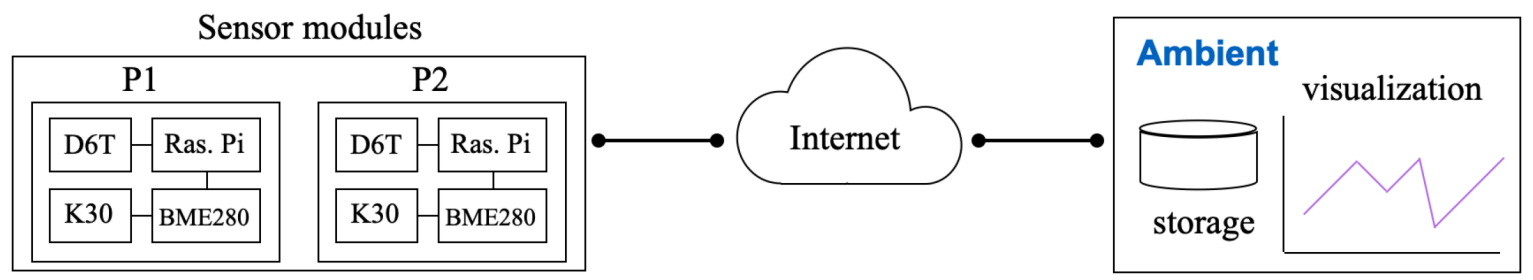

(a)

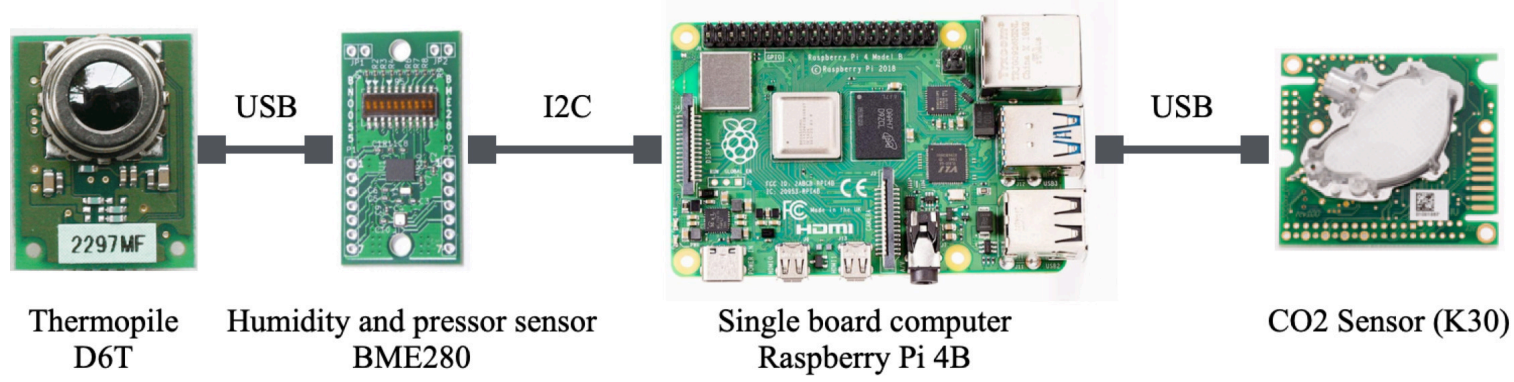

(b)
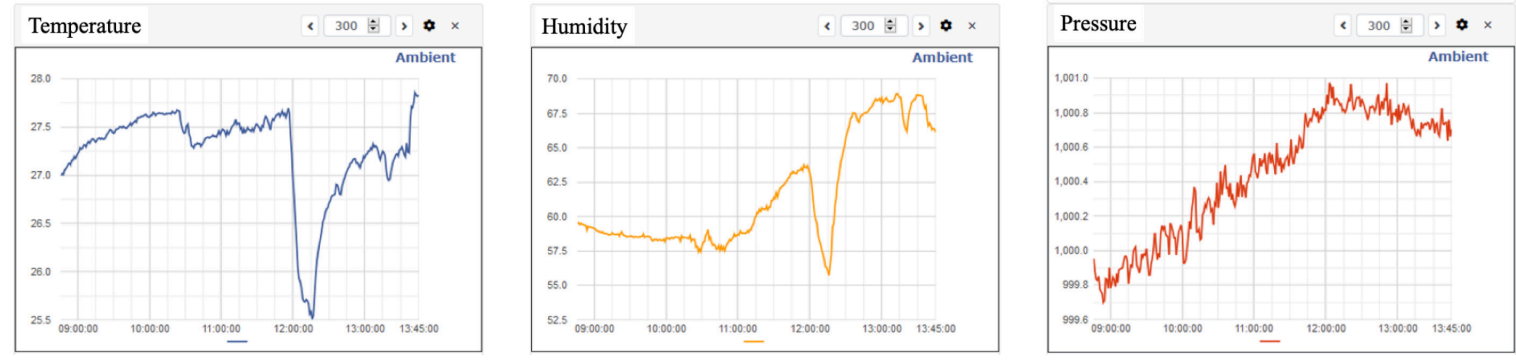

(c)

Figure 6. Overall system architecture of the proposed privacy-aware environmental sensor system. (a) System configuration. (b) Sensor boards and SBC in the sensor module. (c) Images of IoT cloud service Ambient for storage and visualization.

Figure $6 \mathrm{~b}$ depicts details of the sensor module structure. Each sensor module comprises an SBC, which is used for sensor control, data storage, and wireless communication with an Ambient server, along with three sensor boards: a thermopile sensor board (D6T44L-06; Omron Corp.; Kyoto, Japan), a $\mathrm{CO}_{2}$ sensor board (K30; Senseair AB; Delsbo, 
Sweden), and an air pressure, humidity, and temperature combined sensor board (BME280; Robert Bosch GmbH; Gerlingen, Germany). The SBC and these three sensor boards are wired by serial cables with serial communication protocols managed using a universal asynchronous receiver transmitter (UART) and an inter-integrated circuit (I2C). Figure $6 \mathrm{c}$ depicts images of data visualization results on browsers of a website provided by Ambient. We checked the sensor operating status periodically through the website during examination for data collection.

Table 11 presents some major specifications of a thermopile sensor, D6T, which measures the temperature distribution of objects, especially for humans [218], and their surroundings. As a contactless sensor, the D6T sensor measures surface temperatures using thermocouple elements that receive radiant heat energy from objects and surroundings.

Table 11. Major specifications of D6T-44L-06.

\begin{tabular}{cc}
\hline Item & Specifications \\
\hline Number of elements & $16(4 \times 4)$ \\
Horizontal view angle & $44.2^{\circ}$ \\
Vertical view angle & $45.7^{\circ}$ \\
Object temperature detection range & $5-50^{\circ}$ \\
Ambient temperature detection range & $5-45^{\circ}$ \\
Object temperature output accuracy & $\pm 1.5^{\circ}($ maximum $)$ \\
Temperature resolution & $0.06{ }^{\circ} \mathrm{C}$ \\
Operating temperature & $0-50{ }^{\circ} \mathrm{C}$ \\
Operating humidity & $20-95 \%$ \\
Sensor board dimensions & L11.6 $\times \mathrm{W} 12.0 \times \mathrm{H} 10.7 \mathrm{~mm}$ \\
\hline
\end{tabular}

Table 12 presents some major specifications of a $\mathrm{CO}_{2}$ sensor: $\mathrm{K} 30$. The operating principle of this sensor is a non-dispersive infrared (NDIR) technology based on microelectromechanical systems (MEMS) for a small, inexpensive, and lightweight gas sensor. We calibrated this sensor in our earlier study aimed at in-situ atmospheric measurements [219].

Table 12. Major specifications of K30.

\begin{tabular}{cc}
\hline Item & Specifications \\
\hline Target gas & $\mathrm{CO}_{2}$ \\
Operating principle & $\mathrm{NDIR}$ \\
Measurement range & $0-5000 \mathrm{ppm}$ \\
Accuracy & $\pm 30 \mathrm{ppm} \pm 3 \%$ of reading at $101.3 \mathrm{kPa}$ \\
Response time & $20 \mathrm{~s}$ diffusion time \\
Rate of measurement & $0.5 \mathrm{~Hz}$ \\
Operating temperature & $0-50{ }^{\circ} \mathrm{C}$ \\
Operating humidity & $0-95 \%$ \\
Sensor board dimensions & $\mathrm{L} 51 \times \mathrm{W} 58 \times \mathrm{H} 12 \mathrm{~mm}$ \\
\hline
\end{tabular}

Table 13 presents some major specifications of an integrated sensor; BME280, which comprises an air pressure sensor, a humidity sensor, and a temperature sensor as a single sensor package. The measurement ranges for air pressure, temperature, humidity are, respectively, $300-1100 \mathrm{hPa},-40-85{ }^{\circ} \mathrm{C}$, and $0-100 \%$ with $0.18 \mathrm{hPa}, 0.01{ }^{\circ} \mathrm{C}$, and $0.008 \%$ resolutions. 
Table 13. Major specifications of BME280.

\begin{tabular}{ccc}
\hline Item & Target & Specifications \\
\hline Measurement range & Air pressure & $300-1100 \mathrm{hPa}$ \\
& Temperature & $-40-85^{\circ} \mathrm{C}$ \\
& Humidity & $0-100 \%$ \\
Resolution & Air pressure & $0.18 \mathrm{hPa}$ \\
& Temperature & $0.01^{\circ} \mathrm{C}$ \\
Accuracy tolerance & Humidity & $0.008 \%$ \\
& Air pressure & $\pm 1 \mathrm{hPa}$ \\
& Temperature & $\pm 1 \%$ \\
Sampling intervals & Humidity & $\pm 3 \%$ \\
Sensor dimensions & & $1 \mathrm{~s}$ \\
\hline
\end{tabular}

Based on our earlier study [219] and existing studies as shown in Table 2, we used LSTM embedded in VGG-16. Figure 7 portrays a typical LSTM network architecture of hidden layers. The internal LSTM structure comprises hidden layer units with memory cells called LSTM blocks, along with gates of three types: input gates, forgetting gates, and output gates. The vanishing gradient problem [220] is resolved using this mechanism. Input gates select enabled or disabled input feature signals. Forgetting gates select permission to reset the internal information stored in cells. Output gates determine the amount of information that is transmitted at the next phase. Based on recurrent neural network (RNN) algorithms, LSTM provides a one-step later prediction. The prediction is conducted from the input feature signals at the current time $t$ and the feedback signals to the hidden layer at a previous time $t-1$. The memory cells save internal information for a long period, which provides an important benefit compared to RNN for modeling temporally distant dependences.

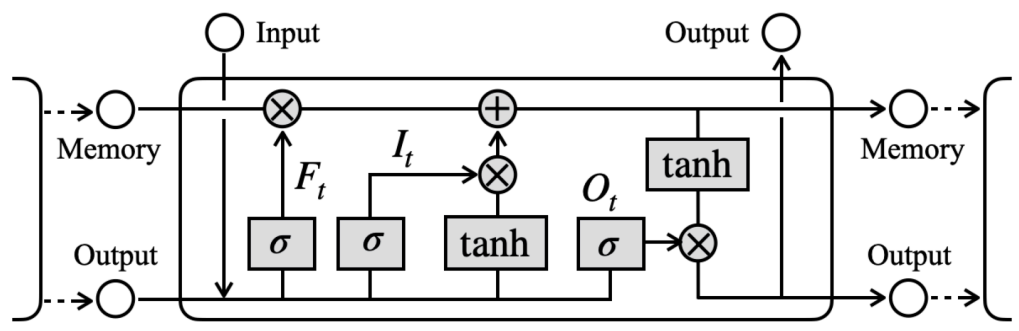

Figure 7. LSTM network architecture of the hidden layer. Signals progress from left to right.

Letting $x_{t}$ and $c_{t}$, respectively, represent the input feature signal and output from memory cells, and letting $I_{t}, F_{t}$, and $O_{t}$, respectively, stand for the outputs of the input, forgetting, and output gates, then LSTM output $H_{t}$ is obtained as presented below.

$$
\begin{gathered}
H_{t}=o_{t} \otimes \tanh \left(c_{t}\right), \\
c_{t}=c_{t-1} \otimes f_{t}+i_{t} \otimes \tanh \left(W_{z} x_{t}+R_{z} h_{t-1}+b_{z}\right), \\
{\left[\begin{array}{c}
I_{t} \\
F_{t} \\
O_{t}
\end{array}\right]=\sigma_{1}\left[\begin{array}{l}
W_{i} \\
W_{f} \\
W_{o}
\end{array}\right] x_{t}+\sigma_{2}\left[\begin{array}{l}
R_{i} \\
R_{f} \\
R_{o}
\end{array}\right] h_{t-1}+\sigma_{3}\left[\begin{array}{l}
b_{i} \\
b_{f} \\
b_{o}
\end{array}\right] .}
\end{gathered}
$$

where $W_{i, f, o, z}, R_{i, f, o, z}$, and $B_{i, f, o, z}$, respectively, stand for input weights, recurrent weights, and biases. Moreover, $\sigma=\sigma_{1}=\sigma_{2}=\sigma_{3}$ and $\otimes$, respectively, express the sigmoid function and the element-wise product.

The dominant role of $I_{t}$ is to update cell states. Propagation signals are controlled by $F_{t}$, which refers to a previous cell state $c_{t-1}$. Moreover, unnecessary signals are removed by $F_{t}$, which prevents excessive information from the earlier cell output split by short- 
term and long-term memories. The output gates control update values from hidden units. Similarly to the input gates, the output gates have a mechanism that is useful in avoiding inappropriate weight updates for redundant and undesired signals. In addition, the current cell state $c_{t}$ and $H_{t}$ are also used to calculate the subsequent input data at $t+1$. As a remarkable characteristic, LSTM has a dynamic adjustment mechanism that provides previous and forward signals while maintaining $c_{t}$ in addition to $H_{t}$.

Table 14 presents the major parameters of LSTM. Based on our earlier study [219], the numbers of generations, batch size, and validation split were set, respectively, to 100 epochs, 2, and 0.2. Each unit of the input layer was assigned to each sensor channel. The number of the input layer comprises two types: 20 units for the single sensor system and 40 units for the dual sensor system. The numbers of the hidden layer and the output layer were set, respectively, to 50 units and 8 units. Similar to the previous experiment, we used Adam [213] for the optimization algorithm between the LSTM layer and the output layer. Finally, we evaluated the look-back parameter, which represents the number of previous time steps to be considered as input, by changing it to three steps: 5, 10, 20, and 30. The optimal value for this parameter was 10.

Table 14. LSTM parameters and their setting values.

\begin{tabular}{cc}
\hline Parameters & Setting Values \\
\hline Learning iteration & 50 epochs \\
Batch size & 2 \\
Validation split & 0.2 \\
Num. input layer & 20 or 40 units \\
Num. hidden layer & 50 units \\
Num. output layer & 8 units \\
Optimization algorithms & Adam [213] \\
Look-back & 10 \\
\hline
\end{tabular}

\subsection{Setup and the Obtained Second Dataset}

We obtained another benchmark dataset using our originally developed sensor system, as depicted in Figure 6. Figure 8a depicts the room layout used in this experiment. The room is $8 \mathrm{~m}$ long and $3 \mathrm{~m}$ wide, with an area of approximately $24 \mathrm{~m}^{2}$. This room is used mainly for a meeting as our satellite laboratory. The two desks have different orientations. We installed the respective sensor modules on the walls at the centers of the longitudinal and latitudinal positions. The installation height was approximately $2.5 \mathrm{~m}$ from the floor. Figure $8 \mathrm{~b}$ depicts an image after sensor installation on the wall.

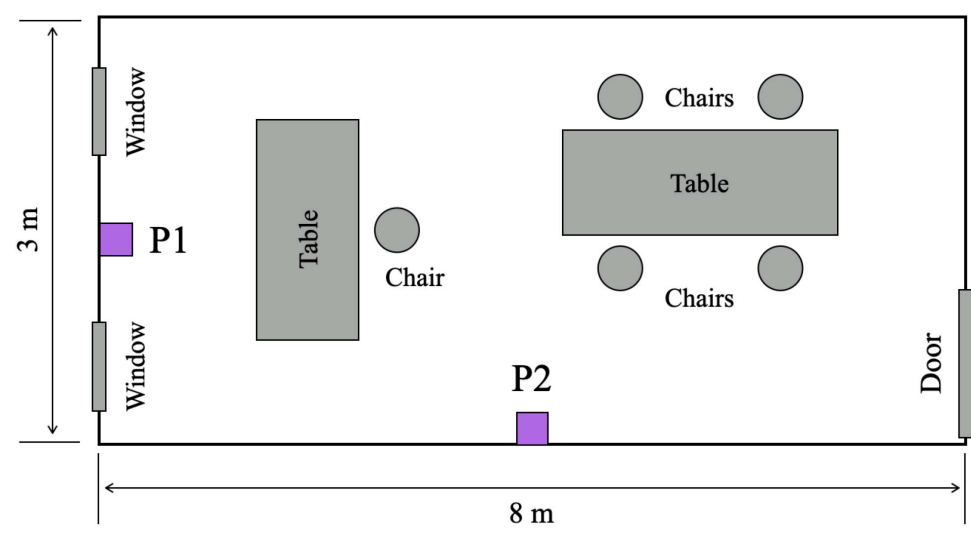

(a) room layout

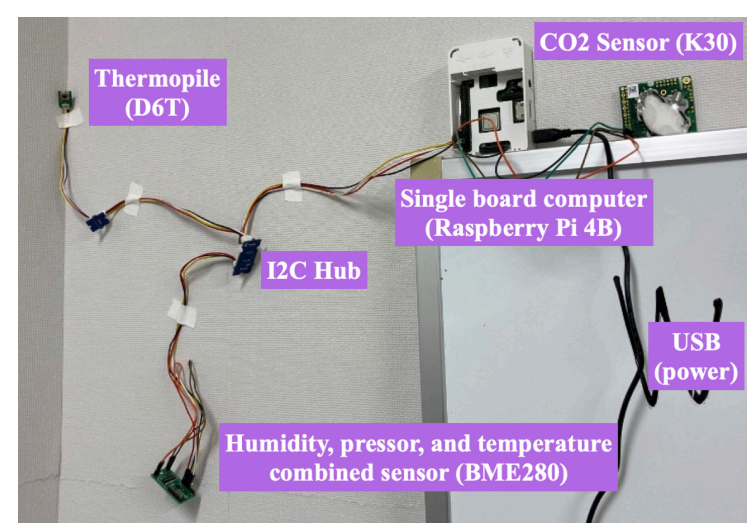

(b) installed sensors

Figure 8. Experiment environment and sensor installation. 
We obtained time-series images using a hemispheric camera (PIXPRO SP360; Eastman Kodak Co., Rochester, NY, USA) for annotation. We used our proposed and evaluated method in Section 3 as a semi-automatic annotation method for labeling behavior patterns for this dataset. The behavior patterns comprise eight categories: eating (ET), operating a laptop computer (LC), operating a smartphone (SP), playing a game (GM), reading (RD), exiting the room (EX), taking a nap (NP), and sitting (ST). Of these categories, ET, LC, SP, RD, and ST are similar to the first dataset. We added GM, EX, and NP as new categories in the second dataset. We annotated them manually.

Table 15 presents the number of signals in each behavior pattern. The total data amount is 11,521 signals. The quantities of data in each behavior are uneven because the subject spent with no restrictions in the room during the data recording. The number of GM data is the largest, accounting for one-third. The numbers of EX, NP, and ST data are small.

Table 15. Details of datasets for respective behavior patterns.

\begin{tabular}{cccccccccc}
\hline & ET & LC & SP & GM & RD & EX & NP & ST & Total \\
\hline No. Signals & 655 & 3142 & 1139 & 3951 & 1444 & 408 & 414 & 368 & 11521 \\
Ratio (\%) & 5.69 & 27.27 & 9.89 & 34.29 & 12.53 & 3.54 & 3.59 & 3.19 & 100.00 \\
\hline
\end{tabular}

\subsection{Experiment Results and Discussion}

Time-series split cross-validation (TSSCV) was used to evaluate the recognition accuracy of behavior patterns. The number of divisions $k$ is the most important parameter for TSSCV. We set $k=8$ as a result based on our preliminary experiment. Figure 9 depicts the distributions of recognition accuracies in each validation result. The distributions demonstrate that the difference in recognition accuracy is greater in each validation data subset.

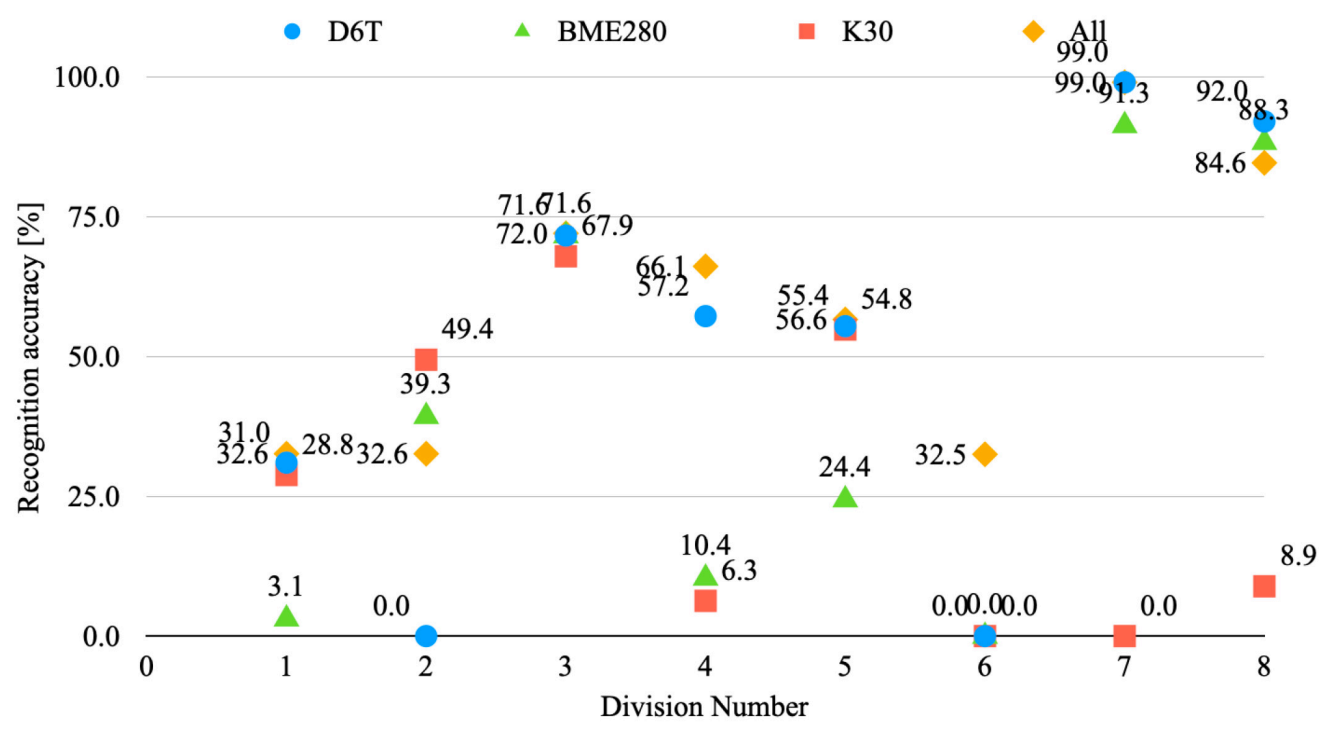

Figure 9. Transition of recognition accuracies with time-series split cross-validation (TSSCV).

Table 16 presents the mean recognition accuracies calculated from the result portrayed in Figure 9. The mean recognition accuracy and its standard deviation from all sensors of both modules are $60.8 \%$ and $12.0 \%$, respectively. Reflecting the performance of the sensor module separately, the third and fourth rows in the table, respectively, show recognition accuracies for P1 or P2. Although the accuracy for P1 dropped 18.1\%, the accuracy drop for $\mathrm{P} 2$ was 3.1\%. This accuracy difference demonstrates that the effect of improved recognition accuracy of P2 was higher than that of P1. For the rectangular room depicted in Figure $8 \mathrm{a}$, the sensor installation on the longitudinal side was more effective than that on the latitudinal side. 
Table 16. Mean recognition accuracies and standard deviations in the respective positions and sensors (\%). Underlining shows the highest accuracy in each sensor combination.

\begin{tabular}{ccccc}
\hline Sensor Installed Position & All Sensors & D6T & BME280 & K30 \\
\hline P1+P2 & $\underline{60.8}(12.0)$ & $\underline{50.6}(17.7)$ & $44.6(17.7)$ & $27.0(12.7)$ \\
P1 & $59.8(16.8)$ & $41.6(17.7)$ & $30.8(16.3)$ & $18.2(9.7)$ \\
P2 & $58.9(13.1)$ & $49.0(16.2)$ & $\underline{46.5}(15.6)$ & $\underline{46.1}(18.5)$ \\
\hline
\end{tabular}

Subsequently, the third, fourth, and fifth columns of Table 16, respectively, present the recognition accuracies for the sole use of either D6T, BME280, or K30. Compared with the three sensors, D6T and K30, respectively, produced $50.6 \%$ as the highest accuracy and $18.2 \%$ as the lowest accuracy. This revealed tendency demonstrates that delay time gaps of measured feature changes affected feature changes of the measurement object. Moreover, these results demonstrate that feature dimensional differences of the respective sensors, such as 16-dimensional signals from D6T, 3D signals from BME280, and one-dimensional (1D) signals from K30, affected the recognition accuracy.

Figure 10 depicts recognition accuracies in each behavior pattern and sensor module. Among the eight behavior patterns, ET exhibited the highest accuracy of more than $90 \%$. Subsequently, LC and GM demonstrated relatively high accuracies: 77.6\%, 54.0\%, 57.6\%, $69.0 \%, 61.3 \%$, and $77.9 \%$. However, the accuracy difference can be large depending on the installation positions of the sensor modules. The accuracies of RD and EX are low compared with those of ET, LC, and GM. For ST, the accuracies of P1+P2, P1, and P2 are, respectively, $2.8 \%, 0.0 \%$, and $0.0 \%$. The respective accuracies of SP and NP are $0.0 \%$. We analyzed these unbalanced results using confusion matrices.

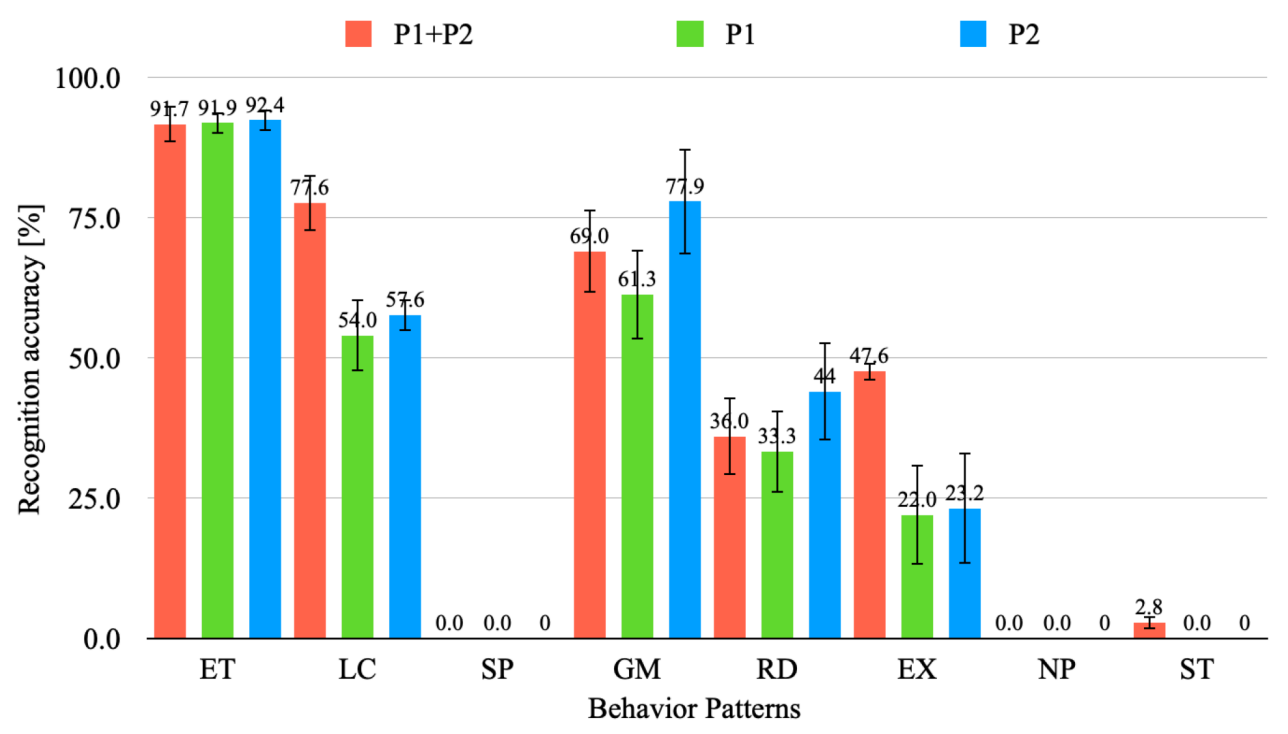

Figure 10. Recognition accuracies of the respective behavior patterns and sensor module.

Tables 17 present confusion matrices. These matrices demonstrated that EX was hindered by some slight confusion with LC in all combinations of the sensor module installation patterns. By contrast, numerous LC signals were confused with RD signals. Particularly, marked confusion occurred between LC and SP for P2 compared with those for P1+P2 and P1. For SP, confusion occurred in all categories, except for EX and ST. For $\mathrm{GM}$, confusion among LC, RD, and NP occurred frequently. Confusion with the other categories occurred less. Although confusion among ET, SP, and ST occurred, the other categories showed less confusion. Numerous instances of confusion occurred between EX and LC. This tendency differs from the confusion property for LC, which caused a few instances of confusion against EX. 
Table 17. Confusion matrices for the results shown in Figure 10. Underlined values are the maximum numbers of signals in the respective recognition categories.

\begin{tabular}{ccccccccc}
\hline P1+P2 & EX & LC & SP & GM & RD & EX & NP & ST \\
\hline EX & 408 & 23 & 4 & 0 & 0 & 10 & 0 & 0 \\
LC & 0 & $\underline{2437}$ & 0 & 62 & 552 & 73 & 0 & 17 \\
SP & 8 & $\underline{51}$ & 0 & 11 & 23 & 0 & 22 & 0 \\
GM & 0 & 439 & 1 & $\underline{2725}$ & 486 & 28 & 270 & 2 \\
RD & 536 & 0 & 468 & 0 & $\underline{627}$ & 0 & 0 & 113 \\
EX & 0 & $\underline{217}$ & 0 & 0 & 0 & 197 & 0 & 0 \\
NP & 0 & 0 & 0 & 0 & $\underline{408}$ & 0 & 0 & 0 \\
ST & 42 & $\underline{214}$ & 0 & 28 & 13 & 16 & 0 & 9 \\
\hline P1 & EX & LC & SP & GM & RD & EX & NP & ST \\
\hline EX & $\underline{409}$ & 23 & 3 & 0 & 0 & 10 & 0 & 0 \\
LC & 0 & $\underline{1696}$ & 0 & 114 & 1324 & 7 & 0 & 0 \\
SP & 8 & $\underline{62}$ & 0 & 13 & 32 & 0 & 0 & 0 \\
GM & 0 & 889 & 0 & $\underline{2420}$ & 642 & 0 & 0 & 0 \\
RD & 463 & 0 & 471 & 0 & $\underline{481}$ & 0 & 0 & 29 \\
EX & 0 & $\underline{323}$ & 0 & 0 & 0 & 91 & 0 & 0 \\
NP & 0 & 0 & 0 & 0 & $\underline{408}$ & 0 & 0 & 0 \\
ST & 42 & $\underline{222}$ & 9 & 28 & 13 & 8 & 0 & 0 \\
\hline P2 & EX & LC & SP & GM & RD & EX & NP & ST \\
\hline EX & $\underline{411}$ & 23 & 1 & 0 & 0 & 10 & 0 & 0 \\
LC & 42 & $\underline{1809}$ & 849 & 81 & 349 & 7 & 0 & 4 \\
SP & 8 & $\underline{51}$ & 0 & 25 & 31 & 0 & 0 & 0 \\
GM & 0 & 390 & 0 & $\underline{3078}$ & 483 & 0 & 0 & 0 \\
RD & 458 & 0 & 351 & 0 & $\underline{635}$ & 0 & 0 & 0 \\
EX & 0 & $\underline{318}$ & 0 & 0 & 0 & 96 & 0 & 0 \\
NP & 0 & 0 & 0 & 0 & $\underline{408}$ & 0 & 0 & 0 \\
ST & 42 & 80 & 9 & 28 & $\underline{155}$ & 8 & 0 & 0 \\
\hline & & & & & & & & \\
\hline
\end{tabular}

All the NP signals were recognized to RD. We consider that both characteristics were less similar because no confusion occurred from RD to NP. Experiments must be conducted to compare these results with those obtained using BLSTM [154] because temporal characteristics are assumed to have some influence. For ST, confusion with categories, except NP, occurred widely. Particularly, numerous ST signals were confused with LD and RD. The recognition output to the LC was large, approximately one-third of the total. Subsequently, GM and RD include numerous signals. SP, EX, NP, and ST are few: less than $5 \%$. As presented in Table 15, GM accounts for the largest number of signals. This result demonstrated that the amount of data does not affect the recognition result distribution. We infer that the low accuracy of SP, EX, NP, and ST is attributable to the small amount of data. However, correlation between the number of data and the obtained accuracy is not a dominant factor because ET represents an exception to the overall tendencies found in this study.

\section{Conclusions}

As a feasibility study based on findings of an HAR mini-survey, this paper presented two original benchmark datasets used to recognize daily life behavior patterns from the assessment of tiny sensor signals generated from slight motions. The first dataset comprises feature vectors from time-series panoramic images obtained using a fisheye lens camera. We used OpenPose and CNN with the VGG-16 backbone to recognize behavior patterns of five types after first dividing them into learning and verification subsets. Our originally obtained first dataset comprises five categories: eating, reading, operating a smartphone, operating a personal computer, and sitting. Recognition evaluation experiments were 
conducted by changing the combination of subsets. The obtained results demonstrated characteristics of behavioral pattern recognition obtained from wide-field images taken using a fisheye lens camera, even for panoramic images that included distortions.

The second dataset was obtained using five environmental sensors. We were concerned not only with non-constraint but also with privacy awareness for this environmental sensor system. Using LSTM combined with CNN, we attempted to recognize eight behavior patterns. Comparing these datasets, the recognition accuracy of the second dataset was 38.9 percentage points lower than that of the first dataset. Nevertheless, we demonstrated the possibility of recognizing behavior patterns from time-series of weak sensor signals. We consider that the recognition results obtained from the first dataset after the accuracy evaluation can be reused for automatic annotation of labels to the second benchmark dataset.

As a subject of future work, we must develop an integrated system that is able to use the two datasets for automatic annotation and autonomous learning without using manually created labels. The limitation of this study is that respective dataset involve only one subject. We intend to develop a dataset accumulated from multiple subjects. Furthermore, intend to expand the categories of target behavior patterns, including data types and volumes. Moreover, our future studies are expected to clarify relations between emotions and behavior patterns through field tests conducted in unusual living environments such as accommodation facilities.

Author Contributions: Conceptualization, H.M.; methodology, H.M.; software, S.N.; validation, H.W.; formal analysis, H.W.; investigation, H.M.; resources, S.N.; data curation, S.N.; writingoriginal draft preparation, H.M.; writing — review and editing, S.N.; visualization, H.W.; supervision, K.S.; project administration, K.S.; funding acquisition, H.M. All authors have read and agreed to the published version of the manuscript.

Funding: This research was supported by the Suzuki Foundation (Tokyo, Japan).

Institutional Review Board Statement: Not applicable.

Informed Consent Statement: Informed consent was obtained from all subjects involved in the study.

Data Availability Statement: Datasets described as a result of this study are available on request to the corresponding author.

Acknowledgments: We would like to express our appreciation to Mimori Kamiyama and Yuuki Sugizaki, who are graduates of Akita Prefectural University, for their great cooperation with experiments.

Conflicts of Interest: The authors declare that they have no conflict of interest. The funders had no role in the design of the study, in the collection, analyses, or in interpretation of data, in the writing of the manuscript, or in the decision to publish the results.

\section{Abbreviations}

The following abbreviations are used in this manuscript:

1D one-dimensional

2D two-dimensional

3D three-dimensional

ADL activities of daily living

$\mathrm{COCO}$ common objects in context

DL deep learning

CML conventional machine learning

CNN convolutional neural network

DC deep clustering

DT decision tree

EBT ensemble bagged trees

EL ensemble learning 


\begin{tabular}{|c|c|}
\hline ELM & extreme learning machine \\
\hline ES & environmental sensors \\
\hline ET & eating \\
\hline EX & exiting \\
\hline FoV & field of view \\
\hline GM & game \\
\hline GPU & graphics processing unit \\
\hline $\mathrm{I} 2 \mathrm{C}$ & inter-integrated circuit \\
\hline IEMO-CAP & interactive emotional dyadic motion capture \\
\hline IoT & internet of things \\
\hline IPL-JPDA & improved pseudo-labels joint probability domain adaptation \\
\hline$k \mathrm{NN}$ & $k$ nearest neighbor \\
\hline LC & laptop computer \\
\hline LDA & linear discriminant analysis \\
\hline LiDAR & light detection and ranging \\
\hline LSTM & short-term memory \\
\hline MCB & multi-channel bidirectional \\
\hline MEMS & microelectromechanical systems \\
\hline MLP & multilayer perceptron \\
\hline MPII & Max Planck Institut Informatik \\
\hline NB & naive Bayes \\
\hline NP & nap \\
\hline NDIR & non-dispersive infrared sensor \\
\hline OME & one-minute-gradual \\
\hline PAFs & part affinity fields \\
\hline PIR & pyroelectric infrared \\
\hline RD & reading \\
\hline RF & random forests \\
\hline RGB-D & red, green, and blue-depth \\
\hline RNN & recurrent neural network \\
\hline $\mathrm{I} 2 \mathrm{C}$ & inter-integrated circuit \\
\hline SBC & single board computer \\
\hline SP & smartphone operation \\
\hline ST & sitting \\
\hline SVM & support vector machines \\
\hline TOF & time of flight \\
\hline TSSCV & time-series split cross-validation \\
\hline TV & television \\
\hline UART & universal asynchronous receiver-transmitter \\
\hline WS & wearable sensors \\
\hline YOLO & you look only once \\
\hline ZSL & zero-shot learning \\
\hline
\end{tabular}

\section{References}

1. Moeslund, T.B.; Hilton, A.; Krüger, V. A Survey of Advances in Vision-Based Human Motion Capture and Analysis. Comput. Vis. Image Underst. 2006, 104, 90-126. [CrossRef]

2. Escalera, S.; Athitsos, V.; Guyon, I. Challenges in Multi-modal Gesture Recognition. Gesture Recognit. 2017, 1-60. doi: 10.1007/9783-319-57021-1_1 [CrossRef]

3. Liu, J.; Gu, Y.; Kamijo, S. Customer Behavior Classification Using Surveillance Camera for Marketing. Multimed. Tools Appl. 2017 76, 6595-6622. [CrossRef]

4. Kanda, T.; Miyashita, T.; Osada, T.; Haikawa, Y.; Ishiguro, H. Analysis of Humanoid Appearances in Human-Robot Interaction. IEEE Trans. Robot. 2008, 24, 725-735. [CrossRef]

5. Shamsuddin, S.; Yussof, H.; Ismail, L.; Hanapiah, F.A.; Mohamed, S.; Piah, H.A.; Zahari, N.I. Initial Response of Autistic Children in Human-Robot Interaction Therapy with Humanoid Robot nao. In Proceedings of the IEEE Eighth International Colloquium on Signal Processing and its Applications, Malacca, Malaysia, 23-25 March 2012; pp. 188-193.

6. Thomas, G.; Gade, R.; Moeslund, T.B.; Carr, P.; Hilton, A. Computer Vision for Sports: Current Applications and Research Topics. Comput. Vis. Image Underst. 2017, 159, 3-18. [CrossRef] 
7. Liu, J.N.K.; Wang, M.; Feng, B. iBotGuard: An Internet-based Intelligent Robot security system using Invariant Face Recognition Against Intruder. IEEE Trans. Syst. Man Cybern. Part C 2005, 35, 97-105. [CrossRef]

8. Bowyer, K.W.; Hollingsworth, K.; Flynn, P.J. Image Understanding for Iris Biometrics: A Survey. Comput. Vis. Image Underst. 2008, 110, 281-307. [CrossRef]

9. Pantic, M.; Rothkrantz, L.J.M. Automatic analysis of facial expressions: The state-of-the art. IEEE Trans. Pattern Anal. Mach. Intell. 2000, 22, 1424-1445. [CrossRef]

10. Hu, W.; Tan, T.; Wang, L.; Maybank, S. A Survey on Visual Surveillance of Object Motion and Behaviors. IEEE Trans. Syst. Man Cybern. Part C 2004, 34, 334-352. [CrossRef]

11. Inoue, M.; Taguchi, R.; Umezaki, T. Vision-based Bed Detection for Hospital Patient Monitoring System. In Proceedings of the 40th Annual International Conference of the IEEE Engineering in Medicine and Biology Society, Honolulu, HI, USA, 17-21 July 2018; pp. 5006-5009.

12. Madokoro, H.; Nakasho, K.; Shimoi, N.; Woo, H.; Sato, K. Development of Invisible Sensors and a Machine-Learning-Based Recognition System Used for Early Prediction of Discontinuous Bed-Leaving Behavior Patterns. Sensors 2020, 20, 1415. [CrossRef]

13. Uddin, M.Z.; Khaksar, W.; Torresen, J. Ambient Sensors for Elderly Care and Independent Living: A Survey. Sensors 2018, 18, 2027. [CrossRef]

14. Foerster, F.; Smeja, M.; Fahrenberg, J. Detection of Posture and Motion by Accelerometry: A Validation Study in Ambulatory Monitoring. Comput. Hum. Behav. 1999, 15, 571-583. [CrossRef]

15. Jähne-Raden, N.; Kulau, U.; Marschollek, M.; Wolf, K.H. INBED: A Highly Specialized System for Bed-Exit-Detection and Fall Prevention on a Geriatric Ward. Sensors 2019, 19, 1017. [CrossRef]

16. Erden, F.; Velipasalar, S.; Alkar, A.Z.; Cetin, A.E. Sensors in Assisted Living: A survey of signal and image processing methods IEEE Signal Process. Mag. 2016, 33, 36-44.

17. Komatsu, Y.; Hamada, K.; Notoya, Y.; Kimura, K. Image Recognition Technology that Helps Save Energy in Room Air Conditioners. Hitachi Rev. 2015, 64, 8.

18. Naslund, J.A.; Aschbrenner, K.A.; Bartels, S.J. Wearable devices and smartphones for activity tracking among people with serious mental illness Ment. Health Phys. Act. 2016, 10, 10-17. [CrossRef] [PubMed]

19. Ramos, C.; Augusto, J.C.; Shapiro, D. Ambient Intelligence-The Next Step for Artificial Intelligence. IEEE Intell. Syst. 2008, 23, 15-18. [CrossRef]

20. Acampora, G.; Cook, D.J.; Rashidi, P.; Vasilakos, A.V. A Survey on Ambient Intelligence in Healthcare. Proc. IEEE Inst. Electr. Eng. 2013, 101, 2470-2494. [CrossRef] [PubMed]

21. LeCun, Y.; Bengio, Y.; Hinton, G. Deep learning. Nature 2015, 521, 436-444. [CrossRef] [PubMed]

22. LeCun, Y.; Boser, B.; Denker, J.S.; Henderson, D.; Howard, R.E.; Hubbard, W.; Jackel, L.D. Backpropagation Applied to Handwritten Zip Code Recognition. Neural Comput. 1989, 1, 541-551. [CrossRef]

23. Hochreiter, S.; Schmidhuber, J. Long Short-Term Memory. Neural Comput. 1997, 9, 1735-1780. [CrossRef] [PubMed]

24. Aggarwal, J.K.; Cai, Q. Human motion analysis: A review. Comput. Vis. Image Underst. 1999, 73, 428-440. [CrossRef]

25. Zhang, F.; Niu, K.; Xiong, J.; Jin, B.; Gu, T.; Jiang, Y.; Zhang, D. Towards a Diffraction-based Sensing Approach on Human Activity Recognition. In Proceedings of the ACM on Interactive, Mobile, Wearable and Ubiquitous Technologies (IMWUT), London, UK, 11-13 September 2019; pp. 1-25.

26. Turaga, P.; Chellappa, R.; Subrahmanian, V.; Udrea, O. Machine Recognition of Human Activities: A Survey. IEEE Trans. Circuits Syst. Video Technol. 2008, 18, 1473-1488. [CrossRef]

27. Candamo, J.; Shreve, M.; Goldgof, D.; Sapper, D.; Kasturi, R. Understanding Transit Scenes: A Survey on Human BehaviorRecognition Algorithms. IEEE Trans. Intell. Transp. Syst. 2010, 11, 206-224. [CrossRef]

28. Lara, O.D.; Labrador, M.A. A Survey on Human Activity Recognition using Wearable Sensors. IEEE Commun. Surv. Tutor. 2013, 15, 1192-1209. [CrossRef]

29. Aggarwal, J.K.; Ryoo, M.S. Human activity analysis: A review. ACM Comput. Surv. 2011, 16, 1-43. [CrossRef]

30. Ke, S.R.; Thuc, H.L.U.; Lee, Y.J.; Hwang, J.N.; Yoo, J.H.; Choi, K.H. A Review on Video-Based Human Activity Recognition. Computers 2013, 2, 88-131. [CrossRef]

31. Aggarwal, J.K.; Xia, L. Human activity recognition from 3D data: A review. Pattern Recognit. Lett. 2014, 48, 70-80. [CrossRef]

32. Dai, J.; Wu, J.; Saghafi, B.; Konrad, J.; Ishwar, P. Towards Privacy-Preserving Activity Recognition Using Extremely Low Temporal and Spatial Resolution Cameras. In Proceedings of the IEEE Computer Society Conference on Computer Vision and Pattern Recognition (CVPR), Boston, MA, USA, 7-12 June 2015; pp. 68-76.

33. Dargan, S.; Kumar, M.; Ayyagari, M.R.; Kumar, G. A Survey of Deep Learning and Its Applications: A New Paradigm to Machine Learning. Arch. Comput. Methods Eng. 2020, 27, 1071-1092. [CrossRef]

34. Chen J.; Ran, X. Deep Learning With Edge Computing: A Review. Proc. IEEE 2019, 107, 1655-1674. [CrossRef]

35. Haensch, W.; Gokmen, T.; Puri, R. The Next Generation of Deep Learning Hardware: Analog Computing. Proc. IEEE 2019, 107, 108-122. [CrossRef]

36. Biswal, A.; Nanda, S.; Panigrahi, C.R.; Cowlessur, S.K.; Pati, B. Human Activity Recognition Using Machine Learning: A Review Prog. Adv. Comput. Intell. Eng. 2021, 323-333. [CrossRef] 
37. Bouchabou, D.; Nguyen, S.M.; Lohr, C.; LeDuc, B.; Kanellos, I. A Survey of Human Activity Recognition in Smart Homes Based on IoT Sensors Algorithms: Taxonomies, Challenges, and Opportunities with Deep Learning. Sensors 2021, 21, 6037. [CrossRef] [PubMed]

38. Chen, K.; Zhang, D.; Yao, L.; Guo, B.; Yu, Z.; Liu, Y. Deep Learning for Sensor-based Human Activity Recognition: Overview, Challenges, and Opportunities. ACM Comput. Surv. 2021, 54, 1-40. [CrossRef]

39. Mihoub, A. A Deep Learning-Based Framework for Human Activity Recognition in Smart Homes. Mob. Inf. Syst. 2021, 2021, 6961343. [CrossRef]

40. Muralidharan, K.; Ramesh, A.; Rithvik, G.; Prem, S.; Reghunaath, A.A.; Gopinath, M.P. 1D Convolution Approach to Human Activity Recognition Using Sensor Data and Comparison with Machine Learning Algorithms. Int. J. Cogn. Comput. Eng. 2021, 2 , 130-143.

41. Shaikh, M.B.; Chai, D. RGB-D Data-Based Action Recognition: A Review. Sensors 2021, 21, 4246. [CrossRef]

42. Straczkiewicz, M.; James, P.; Onnela, J.-P. A Systematic Review of Smartphone-Based Human Activity Recognition Methods for Health Research. Digit. Med. 2021, 4, 148. [CrossRef]

43. Beddiar, D.R.; Nini, B.; Sabokrou, M. Vision-based human activity recognition: A survey. Multimed. Tools Appl. 2020, 79, 30509-30555. [CrossRef]

44. Carvalho, L.I.; Sofia, R.C. A Review on Scaling Mobile Sensing Platforms for Human Activity Recognition: Challenges and Recommendations for Future Research. IoT 2020, 1, 25. [CrossRef]

45. Dang, L.M.; Min, K.; Wang, H.; Piran, J.; Lee, C.H.; Moon, H. Sensor-based and vision-based human activity recognition: A comprehensive survey. Pattern Recognit. 2020, 108, 107561. [CrossRef]

46. Demrozi, F.; Pravadelli, G.; Bihorac, A.; Rashidi, P. Human Activity Recognition Using Inertial, Physiological and Environmental Sensors: A Comprehensive Survey. IEEE Access 2020, 8, 210816-210836. [CrossRef]

47. Fu, B.; Damer, N.; Kirchbuchner, F.; Kuijper, A. Sensing Technology for Human Activity Recognition: A Comprehensive Survey. IEEE Access 2020, 8, 83791-83820. [CrossRef]

48. Hussain, Z.; Sheng, Q.Z.; Zhang, W.E. A review and categorization of techniques on device-free human activity recognition. J. Netw. Comput. Appl. 2020, 167, 102738. [CrossRef]

49. Jung, I.Y. A review of privacy-preserving human and human activity recognition. Int. J. Smart Sens. Intell. Syst. 2020, 1, 13. [CrossRef]

50. Sherafat, B.; Ahn, C.R.; Akhavian, R.; Behzadan, A.H.; Golparvar-Fard, M.; Kim, H.; Lee, Y.C.; Rashidi, A.; Azar, E.R. Automated Methods for Activity Recognition of Construction Workers and Equipment: State-of-the-Art Review. J. Constr. Eng. Manag. 2020, 146, 03120002. [CrossRef]

51. Dang, L.M.; Piran, M.J.; Han, D.; Min, K.; Moon, H. A Survey on Internet of Things and Cloud Computing for Healthcare. Electronics 2019, 8, 768. [CrossRef]

52. Dhiman, C.; Vishwakarma, D.K. A review of state-of-the-art techniques for abnormal human activity recognition. Eng. Appl. Artif. Intell. 2019, 77, 21-45. [CrossRef]

53. Elbasiony, R.; Gomaa, W. A Survey on Human Activity Recognition Based on Temporal Signals of Portable Inertial Sensors. In Proceedings of the International Conference on Advanced Machine Learning Technologies and Applications (AMLTA2), Cairo, Egypt, 28-30 March 2019; pp. 734-745.

54. Hussain, Z.; Sheng, M.; Zhang, W.E. Different Approaches for Human Activity Recognition: A Survey. arXiv 2019, arXiv:1906.05074.

55. Jobanputra, C.; Bavishi, J.; Doshi, N. Human Activity Recognition: A Survey. Procedia Comput. Sci. 2019, 155, 698-703 [CrossRef]

56. Li, X.; He, Y.; Jing, X. A Survey of Deep Learning-Based Human Activity Recognition in Radar. Remote Sens. 2019, 11, 1068. [CrossRef]

57. Sousa Lima, W.; Souto, E.; El-Khatib, K.; Jalali, R.; Gama, J. Human Activity Recognition Using Inertial Sensors in a Smartphone: An Overview. Sensors 2019, 19, 3213. [CrossRef]

58. Slim, S.O.; Atia, A.; Elfattah, M.M.A.; Mostafa, M.S.M. Survey on Human Activity Recognition based on Acceleration Data. Int. J. Adv. Comput. Sci. Appl. 2019, 10, 84-98. [CrossRef]

59. Wang, J.; Chen, Y.; Hao, S.; Peng, X.; Hu, L. Deep learning for sensor-based activity recognition: A survey. Pattern Recognit. Lett. 2019, 119, 3-11. [CrossRef]

60. Nweke, H.F.; Teh, Y.W.; Al-Garadi, M.A.; Alo, U.R. Deep learning algorithms for human activity recognition using mobile and wearable sensor networks: State of the art and research challenges. Expert Syst. Appl. 2018, 105, 233-261. [CrossRef]

61. Ramamurthy, S.R.; Roy, N. Recent trends in machine learning for human activity recognition-A survey. WIREs Data Min. Knowl. Discov. 2018, 8, e1254. [CrossRef]

62. Shickel, B.; Tighe, P.J.; Bihorac, A.; Rashidi, P. DeepEHR: A survey of recent advances in deep learning techniques for electronic health record (EHR) analysis. IEEE J. Biomed. Health Inform. 2018, 22, 1589-1604. [CrossRef]

63. Wang, P.; Li, W.; Ogunbona, P.; Wan, J.; Escalera, S. RGB-D-based human motion recognition with deep learning: A survey. arXiv 2018, arXiv:1711.08362.

64. Cornacchia, M.; Ozcan, K.; Zheng, Y.; Velipasalar, S. A survey on activity detection and classification using wearable sensors. IEEE Sens. J. 2017, 17, 386-403. [CrossRef] 
65. Chen, C.; Jafari, R.; Kehtarnavaz, N. A survey of depth and inertial sensor fusion for human action recognition. Multimed. Tools Appl. 2017, 76, 4405-4425. [CrossRef]

66. Morales, J.; Akopian, D. Physical activity recognition by smartphones, a survey. Biocybern. Biomed. Eng. 2017, 37, 388-400. [CrossRef]

67. Rault, T.; Bouabdallah, A.; Challal, Y.; Marin, F. A survey of energy-efficient context recognition systems using wearable sensors for healthcare applications. Pervasive Mob. Comput. 2017, 37, 23-44. [CrossRef]

68. Vyas, V.V.; Walse, K.H.; Dharaskar, R.V. A Survey on Human Activity Recognition using Smartphone. Int. J. Adv. Res. Comput. Sci. Manag. Stud. 2017, 5, 118-125.

69. Dawn, D.D.; Shaikh, S.H. A comprehensive survey of human action recognition with spatiotemporal interest point (STIP) detector Vis. Comput. 2016, 32, 289-306. [CrossRef]

70. Onofri, L.; Soda, P.; Pechenizkiy, M.; Iannello, G. A survey on using domain and contextual knowledge for human activity recognition in video streams. Expert Syst. Appl. 2016, 63, 97-111. [CrossRef]

71. Fu, Z.; He, X.; Wang, E.; Huo, J.; Huang, J.; Wu, D. Personalized Human Activity Recognition Based on Integrated Wearable Sensor and Transfer Learning. Sensors 2021, 21, 885. [CrossRef]

72. Gorji, A.; Khalid, H.U.R.; Bourdoux, A.; Sahli, H. On the Generalization and Reliability of Single Radar-Based Human Activity Recognition. IEEE Access 2021, 9, 85334-85349. [CrossRef]

73. Gul, M.A.; Yousaf, M.H.; Nawaz, S.; Ur Rehman, Z.; Kim, H. Patient Monitoring by Abnormal Human Activity Recognition Based on CNN Architecture. Electronics 2020, 9, 1993. [CrossRef]

74. Hussain, T.; Nugent, C.; Moore, A.; Liu, J.; Beard, A. A Risk-Based IoT Decision-Making Framework Based on Literature Review with Human Activity Recognition Case Studies. Sensors 2021, 21, 4504. [CrossRef]

75. Mekruksavanich, S.; Jitpattanakul, A. LSTM Networks Using Smartphone Data for Sensor-Based Human Activity Recognition in Smart Homes. Sensors 2021, 21, 1636. [CrossRef]

76. Mekruksavanich, S.; Jitpattanakul, A. Biometric User Identification Based on Human Activity Recognition Using Wearable Sensors: An Experiment Using Deep Learning Models. Electronics 2021, 10, 308. [CrossRef]

77. Moreira, D.; Barandas, M.; Rocha, T.; Alves, P.; Santos, R.; Leonardo, R.; Vieira, P.; Gamboa, H. Human Activity Recognition for Indoor Localization Using Smartphone Inertial Sensors. Sensors 2021, 21, 6316. [CrossRef] [PubMed]

78. Nafea, O.; Abdul, W.; Muhammad, G.; Alsulaiman, M. Sensor-Based Human Activity Recognition with Spatiotemporal Deep Learning. Sensors 2021, 21, 2141. [CrossRef]

79. Xiao, J.; Chen, L.; Chen, H.; Hong, X. Baseline Model Training in Sensor-Based Human Activity Recognition: An Incremental Learning Approach. IEEE Access 2021, 9, 70261-70272. [CrossRef]

80. Ahmed, N.; Rafiq, J.I.; Islam, M.R. Enhanced Human Activity Recognition Based on Smartphone Sensor Data Using Hybrid Feature Selection Model. Sensors 2020, 20, 317. [CrossRef]

81. Ashry, S.; Ogawa, T. Gomaa, W. CHARM-Deep: Continuous Human Activity Recognition Model Based on Deep Neural Network Using IMU Sensors of Smartwatch. IEEE Sens. J. 2020, 20, 8757-8770. [CrossRef]

82. Debache, I.; Jeantet, L.; Chevallier, D.; Bergouignan, A.; Sueur, C. A Lean and Performant Hierarchical Model for Human Activity Recognition Using Body-Mounted Sensors. Sensors 2020, 20, 3090. [CrossRef]

83. Ehatisham-Ul-Haq, M.; Azam, M.A.; Amin, Y.; Naeem, U. C2FHAR: Coarse-to-Fine Human Activity Recognition With Behavioral Context Modeling Using Smart Inertial Sensors. IEEE Access 2020, 8, 7731-7747. [CrossRef]

84. Ferrari, A.; Micucci, D.; Mobilio, M.; Napoletano, P. On the Personalization of Classification Models for Human Activity Recognition. IEEE Access 2020, 8, 32066-32079. [CrossRef]

85. Hamad, R.A.; Yang, L.; Woo, W.L.; Wei, B. Joint Learning of Temporal Models to Handle Imbalanced Data for Human Activity Recognition. Appl. Sci. 2020, 10, 5293. [CrossRef]

86. Ihianle, I.K.; Nwajana, A.O.; Ebenuwa, S.H.; Otuka, R.I.; Owa, K.; Orisatoki, M.O. A Deep Learning Approach for Human Activities Recognition From Multimodal Sensing Devices. IEEE Access 2020, 8, 179028-179038. [CrossRef]

87. Irvine, N.; Nugent, C.; Zhang, S.; Wang, H.; Ng, W.W.Y. Neural Network Ensembles for Sensor-Based Human Activity Recognition Within Smart Environments. Sensors 2020, 20, 216. [CrossRef]

88. Khannouz, M.; Glatard, T. A Benchmark of Data Stream Classification for Human Activity Recognition on Connected Objects. Sensors 2020, 20, 6486. [CrossRef]

89. Lawal, I.A.; Bano, S. Deep Human Activity Recognition With Localisation of Wearable Sensors. IEEE Access 2020, 8, 155060-155070. [CrossRef]

90. Al Machot, F.R.; Elkobaisi, M.; Kyamakya, K. Zero-Shot Human Activity Recognition Using Non-Visual Sensors. Sensors 2020, 20, 825. [CrossRef] [PubMed]

91. Mukherjee, S.; Anvitha, L.; Lahari, T.M. Human activity recognition in RGB-D videos by dynamic images. Multimed. Tools Appl. Vol. 2020, 79, 19787-19801. [CrossRef]

92. Mutegeki, R.; Han, D.S. A CNN-LSTM Approach to Human Activity Recognition. In Proceedings of the International Conference on Artificial Intelligence in Information and Communication (ICAIIC), Fukuoka, Japan, 19-21 February 2020; pp. 362-366.

93. Pham, C.; Pham, C.; Nguyen-Thai, S.; Tran-Quang, H.; Tran, S.; Vu, H.; Tran, T.H.; Le, T.L. SensCapsNet: Deep Neural Network for Non-Obtrusive Sensing Based Human Activity Recognition. IEEE Access 2020, 8, 86934-86946. [CrossRef] 
94. Popescu, A.C.; Mocanu, I.; Cramariuc, B. Fusion Mechanisms for Human Activity Recognition Using Automated Machine Learning. IEEE Access 2020, 8, 143996-144014. [CrossRef]

95. Qin, Z.; Zhang, Y.; Meng, S.; Qin, Z.; Choo, K.K.R. Imaging and fusing time series for wearable sensor-based human activity recognition. Inf. Fusion 2020, 53, 80-87. [CrossRef]

96. Shrestha, A.; Li, H.; Le Kernec, J.; Fioranelli, F. Continuous Human Activity Classification From FMCW Radar With Bi-LSTM Networks. IEEE Sens. J. 2020, 20, 13607-13619. [CrossRef]

97. Taylor, W.; Shah, S.A.; Dashtipour, K.; Zahid, A.; Abbasi, Q.H.; Imran, M.A. An Intelligent Non-Invasive Real-Time Human Activity Recognition System for Next-Generation Healthcare. Sensors 2020, 20, 2653. [CrossRef] [PubMed]

98. Tanberk, S.; Kilimci, Z.H.; Tükel, D.B.; Uysal, M.; Akyokuş, S. A Hybrid Deep Model Using Deep Learning and Dense Optical Flow Approaches for Human Activity Recognition. IEEE Access 2020, 8, 19799-19809. [CrossRef]

99. Wan, S.; Qi, L.; Xu, X.; Tong, C.; Gu, Z. Deep Learning Models for Real-time Human Activity Recognition with Smartphones. Mob. Netw. Appl. 2020, 25, 743-755. [CrossRef]

100. Wang, L.; Liu, R. Human Activity Recognition Based on Wearable Sensor Using Hierarchical Deep LSTM Networks. Circuits Syst. Signal Process. 2020, 39, 837-856. [CrossRef]

101. Xia, K.; Huang, J.; Wang, H. LSTM-CNN Architecture for Human Activity Recognition. IEEE Access 2020, 8, 56855-56866. [CrossRef]

102. Xu, H.; Li, J.; Yuan, H.; Liu, Q.; Fan, S.; Li, T.; Sun, X. Human Activity Recognition Based on Gramian Angular Field and Deep Convolutional Neural Network. IEEE Access 2020, 8, 199393-199405. [CrossRef]

103. Chung, S.; Lim, J.; Noh, K.J.; Kim, G.; Jeong, H. Sensor Data Acquisition and Multimodal Sensor Fusion for Human Activity Recognition Using Deep Learning. Sensors 2019, 19, 1716. [CrossRef]

104. Concone, F.; Re, G.L.; Morana, M. A Fog-Based Application for Human Activity Recognition Using Personal Smart Devices. ACM Trans. Internet Technol. 2019, 19, 1-20. [CrossRef]

105. Ding, R.; Li, X.; Nie, L.; Li, J.; Si, X.; Chu, D.; Liu, G.; Zhan, D. Empirical Study and Improvement on Deep Transfer Learning for Human Activity Recognition. Sensors 2019, 19, 57. [CrossRef]

106. Ding, J.; Wang, Y. WiFi CSI-Based Human Activity Recognition Using Deep Recurrent Neural Network. IEEE Access 2019, 7, 174257-174269. [CrossRef]

107. Gumaei, A.; Hassan, M.M.; Alelaiwi, A.; Alsalman, H. A Hybrid Deep Learning Model for Human Activity Recognition Using Multimodal Body Sensing Data. IEEE Access 2019, 7, 99152-99160. [CrossRef]

108. Ehatisham-Ul-Haq, M.; Javed, A.; Azam, M.A.; Malik, H.M.A.; Irtaza, A.; Lee, I.H.; Mahmood, M.T. Robust Human Activity Recognition Using Multimodal Feature-Level Fusion. IEEE Access 2019, 7, 60736-60751. [CrossRef]

109. Gani, M.O.; Richard, T.F.; Roger, J.P.; Muhammad, O.S.; Ahmed, A.; Sheikh, J.K.; Ahamed, I. A lightweight smartphone based human activity recognition system with high accuracy. J. Netw. Comput. Appl. 2019, 141, 59-72. [CrossRef]

110. Li, H.; Shrestha, A.; Heidari, H.; Le Kernec, J.; Fioranelli, F. Bi-LSTM Network for Multimodal Continuous Human Activity Recognition and Fall Detection. IEEE Sens. J. 2019, 20, 1191-1201. [CrossRef]

111. Kim, K.; Jalal, A.; Mahmood, M. Vision-Based Human Activity Recognition System Using Depth Silhouettes: A Smart Home System for Monitoring the Residents. J. Electr. Eng. Technol. 2019, 14, 2567-2573. [CrossRef]

112. Naveed, H.; Khan, G.; Khan, A.U. Asad Ullah Khan, Aiman Siddiqi; Muhammad Usman Ghani Khan Human activity recognition using mixture of heterogeneous features and sequential minimal optimization. Int. J. Mach. Learn. Cybern. 2019, 10, $2329-2340$. [CrossRef]

113. Qi, W.; Su, H.; Yang, C.; Ferrigno, G.; De Momi, E.; Aliverti, A. A Fast and Robust Deep Convolutional Neural Networks for Complex Human Activity Recognition Using Smartphone. Sensors 2019, 19, 3731. [CrossRef]

114. Siirtola, P.; Röning, J. Incremental Learning to Personalize Human Activity Recognition Models: The Importance of Human AI Collaboration. Sensors 2019, 19, 5151. [CrossRef] [PubMed]

115. Tian, Y.; Zhang, J.; Chen, L.; Geng, Y.; Wang, X. Single Wearable Accelerometer-Based Human Activity Recognition via Kernel Discriminant Analysis and QPSO-KELM Classifier. IEEE Access 2019, 7, 109216-109227. [CrossRef]

116. Voicu, R.A.; Dobre, C.; Bajenaru, L.; Ciobanu, R.I. Human Physical Activity Recognition Using Smartphone Sensors. Sensors 2019, 19, 458. [CrossRef]

117. Xu, S.; Tang, Q.; Jin, L.; Pan, Z. A Cascade Ensemble Learning Model for Human Activity Recognition with Smartphones. Sensors 2019, 19, 2307. [CrossRef]

118. Xu, C.; Chai, D.; He, J.; Zhang, X.; Duan, S. InnoHAR: A Deep Neural Network for Complex Human Activity Recognition. IEEE Access 2019, 7, 9893-9902. [CrossRef]

119. Yang, Y.; Hou, C.; Lang, Y.; Guan, D.; Huang, D.; Xu, J. Open-set human activity recognition based on micro-Doppler signatures. Pattern Recognit. 2019, 85, 60-69. [CrossRef]

120. Zebin, T.; Scully, P.J.; Peek, N.; Casson, A.J.; Ozanyan, K.B. Design and Implementation of a Convolutional Neural Network on an Edge Computing Smartphone for Human Activity Recognition. IEEE Access 2019, 7, 133509-133520. [CrossRef]

121. Zhang, Y.; Zhang, Z.; Zhang, Y.; Bao, J.; Zhang, Y.; Deng, H. Human Activity Recognition Based on Motion Sensor Using U-Net. IEEE Access 2019, 7, 75213-75226. [CrossRef]

122. Zhu, R.; Xiao, Z.; Li, Y.; Yang, M.; Tan, Y.; Zhou, L.; Lin, S.; Wen, H. Efficient Human Activity Recognition Solving the Confusing Activities Via Deep Ensemble Learning. IEEE Access 2019, 7, 75490-75499. [CrossRef] 
123. Espinilla, M.; Medina, J.; Nugent, C. UCAmI Cup. Analyzing the UJA Human Activity Recognition Dataset of Activities of Daily Living. Proceedings 2018, 2, 1267. [CrossRef]

124. Anguita, D.; Ghio, A.; Oneto, L.; Parra, X.; Reyes-Ortiz, J. Energy efficient smartphone-based activity recognition using fixed-point arithmetic. Int. J. Univers. Comput. Sci. 2013, 19, 1295-1314.

125. Zhang, M.; Sawchuk, A. USC-HAD: A daily activity dataset for ubiquitous activity recognition using wearable sensors. In Proceedings of the ACM Conference on Ubiquitous Computing (UbiComp) Workshop on Situation, Activity and Goal Awareness (SAGAware), Pittsburgh, PA, USA, 5-8 September 2012.

126. Kwapisz, J.R.; Weiss, G.M.; Moore, S.A. Activity recognition using cell phone accelerometers. ACM SigKDD Explor. Newsl. 2011, 12, 74-82. [CrossRef]

127. Reyes-Ortiz, J.L.; Oneto, L.; Samá, A.; Parra, X.; Anguita, D. Transition-Aware Human Activity Recognition Using Smartphones. Neurocomputing; Springer: Berlin/Heidelberg, Germany, 2015.

128. Vaizman, Y.; Ellis, K.; Lanckriet, G.; Weibel, N. ExtraSensory app: Data collection in-the-wild with rich user interface to self-report behavior. In Proceedings of the CHI Conference on Human Factors in Computing Systems, Montréal, QC, Canada, 21-26 April 2018; pp. 1-12.

129. Vavoulas, G.; Chatzaki, C.; Malliotakis, T.; Pediaditis, M.; Tsiknakis, M. The MobiAct dataset: Recognition of activities of daily living using smartphones. In Proceedings of the Information and Communication Technologies for Ageing Well and e-Health (ICT4AWE), Rome, Italy, 21-22 April 2016; pp. 143-151.

130. Micucci, D.; Mobilio, M.; Napoletano, P. UniMiB SHAR: A Dataset for Human Activity Recognition Using Acceleration Data from Smartphones. Appl. Sci. 2017, 7, 1101. doi: 10.3390/app7101101 [CrossRef]

131. Malekzadeh, M.; Clegg, R.G.; Cavallaro, A.; Haddadi, H. Protecting sensory data against sensitive inferences. In Proceedings of the First Workshop on Privacy by Design in Distributed Systems (W-P2DS), Porto, Portugal, 23-26 April 2018; pp. 1-6.

132. Ordonez Morales, F.J.; De Toledo, P.; Sanchis, A. Activity Recognition Using Hybrid Generative/Discriminative Models on Home Environments Using Binary Sensors. Sensors 2013, 13, 5460-5477. [CrossRef]

133. Van Kasteren, T.L.; Englebienne, G.; Kröse, B.J. Human activity recognition from wireless sensor network data: Benchmark and software. In Activity Recognition in Pervasive Intelligent Environments; Springer: Berlin/Heidelberg, Germany, $2011 ;$ pp. 165-186.

134. Kasteren, T.; Englebienne, G.; Kröse, B. An activity monitoring system for elderly care using generative and discriminative models. Pers. Ubiquitous Comput. 2010, 14, 489-498. [CrossRef]

135. Banos, O.; Garcia, R.; Holgado-Terriza, J.A.; Damas, M.; Pomares, H.; Rojas, I.; Saez, A.; Villalonga, C. mHealthDroid: A novel framework for agile development of mobile health applications. In Proceedings of the Sixth International Work-Conference Ambient Assisted Living and Daily Activities (IWAAL), Belfast, UK, 2-5 December 2014; pp. 91-98.

136. Weiss, G.M.; Yoneda, K.; Hayajneh, T. Smartphone and Smartwatch-Based Biometrics Using Activities of Daily Living. IEEE Access 2019, 7, 133190-133202. [CrossRef]

137. Morris, D.; Saponas, T.S.; Guillory, A.; Kelner, I. RecoFit: Using a Wearable Sensor to Find, Recognize, and Count Repetitive Exercises. In Proceedings of the SIGCHI Conference on Human Factors in Computing Systems, Toronto, ON, Canada, 6 April 2014; pp. 3225-3234.

138. Sztyler, T.; Stuckenschmidt, H.; Petrich, W. Position-aware activity recognition with wearable devices. Pervasive Mob. Comput. 2017, 38, 281-295. [CrossRef]

139. Cook, D.J.; Crandall, A.S.; Thomas, B.L.; Krishnan, N.C. CASAS: A Smart Home in a Box. Computer 2013, 46, 62-69. [CrossRef]

140. Li, W.; Zhang, Z.; Liu, Z. Action recognition based on a bag of 3D points. In Proceedings of the IEEE Computer Society Conference on Computer Vision and Pattern Recognition (CVPR), San Francisco, CA, USA, 13-18 June 2010; pp. 9-14.

141. Wang, J.; Liu, Z.; Wu, Y.; Yuan, J. Mining actionlet ensemble for action recognition with depth cameras. In Proceedings of the IEEE Computer Society Conference on Computer Vision and Pattern Recognition (CVPR), Providence, RI, USA, 16-21 June 2012; pp. 1290-1297.

142. Chen, C.; Jafari, R.; Kehtarnavaz, N. UTD-MHAD: A multimodal dataset for human action recognition utilizing a depth camera and a wearable inertial sensor. In Proceedings of the IEEE International Conference on Image Processing (ICIP) Quebec City, QC, Canada, 27-30 September 2015; pp. 168-172.

143. Shahroudy, A.; Liu, J.; Ng, T.T.; Wang, G. NTU RGB+D: A Large Scale Dataset for 3D Human Activity Analysis. In Proceedings of the IEEE Computer Society Conference on Computer Vision and Pattern Recognition (CVPR), Las Vegas, NV, USA, 26 June-1 July 2016; pp. 1010-1019.

144. Stisen, A.; Blunck, H.; Bhattacharya, S.; Prentow, T.S.; Kjaergaard, M.B.; Dey, A.; Sonne, T.; Jensen, M.M. Smart Devices are Different: Assessing and MitigatingMobile Sensing Heterogeneities for Activity Recognition. In Proceedings of the 13th ACM Conference on Embedded Networked Sensor Systems, Seoul, Korea, 1-4 November 2015; pp. 127-140.

145. Reyes-Ortiz, J.L.; Oneto, L.; Samá, A.; Parra, X.; Anguita, D. Transition-Aware Human Activity Recognition Using Smartphones. Neurocomputing 2016, 171, 754-767. [CrossRef]

146. Chavarriaga, R.; Sagha, H.; Calatroni, A.; Digumarti, S.T.; Tröster, G.; Millán, J.D.R.; Roggen, D. The opportunity challenge: A benchmark database for on-body sensor-based activity recognition. Pattern Recognit. Lett. 2013, 34, 2033-2042. [CrossRef]

147. Roggen, D.; Calatroni, A.; Rossi, M.; Holleczek, T.; Förster, K.; Tröster, G.; Lukowicz, P.; Bannach, D.; Pirkl, G.; Ferscha, A.; et al. Collecting complex activity datasets in highly rich networked sensor environments. In Proceedings of the Seventh International Conference on Networked Sensing Systems (INSS), Kassel, Germany, 15-18 June 2010; pp. 233-240. 
148. Altun, K.; Barshan, B.; Tunçel, O. Comparative study on classifying human activities with miniature inertial and magnetic sensors. Pattern Recognit. 2010, 43, 3605-3620. [CrossRef]

149. Banos, O.; Villalonga, C.; Garcia, R.; Saez, A.; Damas, M.; Holgado-Terriza, J.A.; Lee, S.; Pomares, H.; Rojas, I. Design, implementation and validation of a novel open framework for agile development of mobile health applications. Biomed. Eng. Online 2015, 14, S6. [CrossRef]

150. Gorelick, L.; Blank, M.; Shechtman, E.; Irani, M.; Basri, R. Actions as Space-Time Shapes. IEEE Trans. Pattern Anal. Mach. Intell. 2007, 29, 2247-2253. [CrossRef]

151. Schuldt, C.; Laptev, I.; Caputo, B. Recognizing human actions: A local SVM approach. In Proceedings of the 17th International Conference on Pattern Recognition (ICPR), Cambridge, UK, 26-26 August 2004.

152. Reiss, A.; Stricker, D. Introducing a New Benchmarked Dataset for Activity Monitoring. In Proceedings of the 16th International Symposium on Wearable Computers, Newcastle, UK, 18-22 June 2012; pp. 108-109.

153. Shi, X.; Chen, Z.; Wang, H.; Yeung, D.Y.; Wong, W.K.; Woo, W.C. Convolutional LSTM network: A machine learning approach for precipitation nowcasting. In Proceedings of the Advances in Neural Information Processing Systems 28 (NIPS), Montreal, QC, Canada, 7-12 December 2015; pp. 1-9.

154. Schuster, M.; Paliwal, K.K. Bidirectional recurrent neural networks. IEEE Trans. Signal Process. 1997, 45, 2673-2681. [CrossRef]

155. Vapnik, V.N. An overview of statistical learning theory. IEEE Trans. Neural Netw. 1999, 10, 988-999. [CrossRef]

156. Breiman, L. Random Forests. Mach. Learn. 2001, 45, 5-32. [CrossRef]

157. LeCun, Y.; Bottou, L.; Bengio, Y.; Haffner, P. Gradient-based learning applied to document recognition. Proc. IEEE 1998, 86, 2278-2324. [CrossRef]

158. Szegedy, C.; Liu, W.; Jia, Y.; Sermanet, P.; Reed, S.; Anguelov, D.; Erhan, D.; Vanhoucke, V.; Rabinovich, A. Going deeper with convolutions. In Proceedings of the IEEE Computer Society Conference on Computer Vision and Pattern Recognition (CVPR), Boston, MA, USA, 8-10 June 2015; pp. 1-9.

159. Simonyan, K.; Zisserman, A. Very Deep Convolutional Networks for Large-Scale Image Recognition. In Proceedings of the International Conference on Learning Representations, San Diego, CA, USA, 7-9 May 2015.

160. Chollet, F. Xception: Deep Learning With Depthwise Separable Convolutions. In Proceedings of the IEEE Conference on Computer Vision and Pattern Recognition (CVPR), Honolulu, HI, USA, 21-26 July 2017; pp. 1251-1258.

161. He, K.; Zhang, X.; Ren, S.; Sun, J. Deep residual learning for image recognition. In Proceedings of the IEEE Computer Society Conference on Computer Vision and Pattern Recognition (CVPR), Las Vegas, NV, USA, 26 June-1 July 2016; pp.770-778.

162. Redmon, J.; Divvala, S.; Girshick, R.; Farhadi, A. You Only Look Once: Unified, Real-Time Object Detection. In Proceedings of the IEEE Computer Society Conference on Computer Vision and Pattern Recognition (CVPR), Las Vegas, NV, USA, 26 June-1 July 2016; pp. 779-788.

163. Huang, G.B.; Zhu, Q.Y.; Siew, C.K. Extreme learning machine: Theory and applications. Neurocomputing 2006, 70, $489-501$. [CrossRef]

164. Xian, Y.; Lampert, C.H.; Schiele, B.; Akata, Z. Zero-Shot Learning-A Comprehensive Evaluation of the Good, the Bad and the Ugly. IEEE Trans. Pattern Anal. Mach. Intell. 2019, 41, 2251-2265. [CrossRef] [PubMed]

165. Ronneberger, O.; Fischer, P.; Brox, T. U-net: Convolutional networks for biomedical image segmentation. In Proceedings of the International Conference on Medical Image Computing and Computer-Assisted Intervention (MICCAI), Munich, Germany, 5-9 October 2015; pp. 234-241.

166. Anguita, D.; Ghio, A.; Oneto, L.; Parra, X.; Reyes-Ortiz, J.L. A Public Domain Dataset for Human Activity Recognition Using Smartphones. In Proceedings of the Computational Intelligence and Machine Learning (ESANN 2013), Bruges, Belgium, 24-26 April 2013; pp. 437-442.

167. Anguita, D.; Ghio, A.; Oneto, L.; Parra, X.; Reyes-Ortiz, J.L. Human activity recognition on smartphones using a multiclass hardware-friendly support vector machine. In Proceedings of the International Workshop on Ambient Assisted Living (IWAAL), Vitoria-Gasteiz, Spain, 3-5 December 2012; pp. 216-223.

168. Gubbi, J.; Buyya, R.; Marusic, S.; Palaniswami, M. Internet of Things (IoT): A vision, architectural elements, and future directions. Future Gener. Comput. Syst. 2013, 29, 1645-1660. [CrossRef]

169. Subasi, A.; Radhwan, M.; Kurdi, R.; Khateeb, K. IoT based mobile healthcare system for human activity recognition. In Proceedings of the 15th Learning and Technology Conference (L\&T), Jeddah, Saudi Arabia, 25-26 February 2018; pp. 29-34.

170. Jourdan, T.; Boutet, A.; Frindel, C. Toward privacy in IoT mobile devices for activity recognition. In Proceedings of the 15th EAI International Conference on Mobile and Ubiquitous Systems: Computing, Networking and Services (MobiQuitous'18), New York, NY, USA, 5-7 November 2018; pp. 155-165

171. Zhang, H.; Xiao, Z.; Wang, J.; Li, F.; Szczerbicki, E. A Novel IoT-Perceptive Human Activity Recognition (HAR) Approach Using Multihead Convolutional Attention. IEEE Internet Things J. 2020, 7, 1072-1080. [CrossRef]

172. Hendry, D.; Chai, K.; Campbell, A.; Hopper, L.; O'Sullivan, P.; Straker, L. Development of a Human Activity Recognition System for Ballet Tasks. Sports Med. Open 2020, 6, 10. [CrossRef]

173. Ekman, P.; Davidson, R.J. The Nature of Emotion: Fundamental Questions; Oxford University Press: Oxford, UK, 1994.

174. Nguyen, T.H.C.; Nebel, J.C.; Florez-Revuelta, F. Recognition of Activities of Daily Living with Egocentric Vision: A Review. Sensors 2016, 16, 72. [CrossRef] [PubMed] 
175. Wiener, J.M.; Hanley, R.J.; Clark, R.; Van Nostrand, J.F. Measuring the Activities of Daily Living: Comparisons Across National Surveys. J. Gerontol. 1990, 45, 229-237. [CrossRef] [PubMed]

176. Pirsiavash, H.; Ramanan, D. Detecting activities of daily living in first-person camera views. In Proceedings of the IEEE Computer Society Conference on Computer Vision and Pattern Recognition (CVPR), Providence, RI, USA, 16-21 June 2012; pp. $2847-2854$.

177. Chelli, A.; Pátzold, M. A Machine Learning Approach for Fall Detection and Daily Living Activity Recognition. IEEE Access 2019, 7, 38670-38687. [CrossRef]

178. Diraco, G.; Leone, A.; Sicilia, P. AI-Based Early Change Detection in Smart Living Environments. Sensors 2019, 19, 3549. [CrossRef] [PubMed]

179. Busso, C.; Bulut, M.; Lee, C.C.; Kazemzadeh, A.; Mower, E.; Kim, S.; Chang, J.N.; Lee, S.; Narayanan, S.S. IEMOCAP: Interactive Emotional Dyadic Motion Capture Database. Lang. Resour. Eval. 2008, 42, 335. [CrossRef]

180. Zadeh, A.; Zellers, R.; Pincus, E.; Morency, L.P. Mosi: Multimodal Corpus of Sentiment Intensity and Subjectivity Analysis in Online Opinion Videos. IEEE Intell. Syst. 2016, 31, 82-88. [CrossRef]

181. Dhall, A.; Goecke, R.; Ghosh, S.; Joshi, J.; Hoey, J.; Gedeon, T. From individual to group-level emotion recognition: Emotiw 5.0 In Proceedings of the 19th ACM International Conference on Multimodal Interaction (ICMI), Glasgow, Scotland, UK, 13-17 November 2017; pp. 524-528.

182. Mollahosseini, A.; Hasani, B.; Mahoor, M.H. AffectNet: A Database for Facial Expression, Valence, and Arousal Computing in the Wild. IEEE Trans. Affect. Comput. 2019, 10, 18-31. [CrossRef]

183. Ekman, P.; Friesen, W.V. Constants across cultures in the face and emotion. J. Personal. Soc. Psychol. 1971, 17, 124. [CrossRef] [PubMed]

184. Nojavanasghari, B.; Baltrusáitis, T.; Hughes, C.E.; Morency, L.P. EmoReact: A Multimodal Approach and Dataset for Recognizing Emotional Responses in Children. In Proceedings of the 18th ACM International Conference on Multimodal Interaction, Tokyo, Japan, 12-16 November 2016; pp. 137-144.

185. Chen, O.O.R.W.; Picard, R.W. Gifgif+: Collecting emotional animated gifs with clustered multi-task learning. In Proceedings of the Seventh International Conference on Affective Computing and Intelligent Interaction, San Antonio, TX, USA, 23-26 October 2017; pp. 510-517.

186. Kim, Y.; Soyata, T.; Behnagh, R.F. Towards Emotionally Aware AI Smart Classroom: Current Issues and Directions for Engineering and Education. IEEE Access 2018, 6, 5308-5331. [CrossRef]

187. Marinoiu, E.; Zanfir, M.; Olaru, V.; Sminchisescu, C. 3D Human Sensing, Action and Emotion Recognition in Robot-Assisted Therapy of Children With Autism. In Proceedings of the IEEE Computer Society Conference on Computer Vision and Pattern Recognition (CVPR), Salt Lake City, UT, USA, 18-23 June 2018; pp. 2158-2167.

188. Ahmed, F.; Bari, A.S.M.H.; Gavrilova, M.L. Emotion Recognition from Body Motion. IEEE Access 2020, 8, 11761-11781. [CrossRef]

189. Ma, Y.; Paterson, H.M.; Pollick, F.E. A Motion Capture Library for the Study of Identity, Gender, and Emotion Perception from Biological Motion. Behav. Res. Methods 2006, 38, 134-141. [CrossRef]

190. Cao, Z.; Hidalgo, G.; Simon, T.; Wei, S.-E.; Sheikh, Y. OpenPose: Realtime Multi-Person 2D Pose Estimation Using Part Affinity Fields. IEEE Trans. Pattern Anal. Mach. Intell. 2021, 43, 172-186. [CrossRef] [PubMed]

191. Nibali, A.; He, Z.; Morgan, S.; Prendergast, L. 3D Human Pose Estimation With 2D Marginal Heatmaps. In Proceedings of the IEEE Winter Conference on Applications of Computer Vision, Long Beach, CA, USA, 16-20 June 2019; pp. 1477-1485.

192. Sun, K.; Xiao, B.; Liu, D.; Wang, J. Deep High-Resolution Representation Learning for Human Pose Estimation. In Proceedings of the IEEE Computer Society Conference on Computer Vision and Pattern Recognition (CVPR), Long Beach, CA, USA, 16-20 June 2019; pp. 5693-5703.

193. Belagiannis, V.; Zisserman, A. Recurrent Human Pose Estimation. In Proceedings of the 12th IEEE International Conference on Automatic Face and Gesture Recognition (FG), Washington, DC, USA, 30 May-3 June 2017; pp. 468-475.

194. Papandreou, G.; Zhu, T.; Chen, L.C.; Gidaris, S.; Tompson, J.; Murphy, K. PersonLab: Person Pose Estimation and Instance Segmentation with a Bottom-Up, Part-Based, Geometric Embedding Model. In Proceedings of the European Conference on Computer Vision (ECCV), Munich, Germany, 8-14 September 2018; pp. 269-286.

195. Fang, H.S.; Xie, S.; Tai, Y.W.; Lu, C. RMPE: Regional Multi-Person Pose Estimation. In Proceedings of the IEEE Computer Society Conference on Computer Vision and Pattern Recognition (CVPR), Honolulu, HI, USA, 21-26 July 2017; pp. $2334-2343$.

196. Insafutdinov, E.; Pishchulin, L.; Andres, B.; Andriluka, M.; Schiele, B. Deepercut: A Deeper, Stronger, and Faster Multi-Person Pose Estimation Model. In Proceedings of the European Conference on Computer Vision (ECCV), Amsterdam, The Netherlands, 8-16 October 2016; pp. 34-50.

197. Pishchulin, L.; Insafutdinov, E.; Tang, S.; Andres, B.; Andriluka, M.; Gehler, P.; Schiele, B. Deepcut: Joint Subset Partition and Labeling for Multi-Person Pose Estimation. In Proceedings of the IEEE Computer Society Conference on Computer Vision and Pattern Recognition (CVPR), Las Vegas, NV, USA, 26 June-1 July 2016; pp. 4929-4937.

198. Wei, S.E.; Ramakrishna, V.; Kanade, T.; Sheikh, Y. Convolutional Pose Machines. In Proceedings of the IEEE Computer Society Conference on Computer Vision and Pattern Recognition (CVPR), Las Vegas, NV, USA, 26 June-1 July 2016 ; pp. 4724-4732.

199. Carreira, J.; Agrawal, P.; Fragkiadaki, K.; Malik, J. Human Pose Estimation with Iterative Error Feedback. In Proceedings of the IEEE Computer Society Conference on Computer Vision and Pattern Recognition (CVPR), Las Vegas, NV, USA, 26 June-1 July 2016; pp. 4733-4742. 
200. Noori, F.M.; Wallace, B.; Uddin, M.Z.; Torresen, J. A Robust Human Activity Recognition Approach Using OpenPose, Motion Features, and Deep Recurrent Neural Network. In Proceedings of the Scandinavian Conference on Image Analysis (SCIA), Norrköping, Sweden, 11-13 June 2019; pp. 299-310.

201. Kim, W.; Sung, J.; Saakes, D.; Huang, C.; Xiong, S. Ergonomic postural assessment using a new open-source human pose estimation technology (OpenPose). Int. J. Ind. Ergon. 2021, 84, 103164. [CrossRef]

202. Cao, Z.; Simon, T.; Wei, S.-E.; Sheikh, Y. Realtime multi-person $2 \mathrm{~d}$ pose estimation using part affinity fields. In Proceedings of the IEEE Conference on Computer Vision and Pattern Recognition (CVPR), Honolulu, HI, USA, 21-26 July 2017; pp. 7291-7299.

203. Andriluka, M.; Pishchulin, L.; Gehler, P.; Schiele, B. 2D Human Pose Estimation: New Benchmark and State of the Art Analysis. In Proceedings of the IEEE Computer Society Conference on Computer Vision and Pattern Recognition (CVPR), Columbus, OH, USA, 24-27 June 2014; pp. 3686-3693.

204. Iqbaland, U.; Gall, J. Multi-Person Pose Estimation with Local Joint-to-Person Associations. In Proceedings of the European Conference on Computer Vision (ECCV), Amsterdam, The Netherlands, 8-16 October 2016; pp. 627-642.

205. Pan, S.J.; Yang, Q. A Survey on Transfer Learning. IEEE Trans. Knowl. Data Eng. 2010, 22, 1345-1359. [CrossRef]

206. Deng, J.; Dong, W.; Socher, R.; Li, L.; Li, K.; Fei-Fei, L. ImageNet: A Large-Scale Hierarchical Image Database. In Proceedings of the IEEE Computer Society Conference on Computer Vision and Pattern Recognition (CVPR), Miami Beach, FL, USA, 22-24 June 2009; pp. 248-255.

207. Zagoruyko, S.; Komodaki, N. Wide residual networks. arXiv 2016, arXiv:1605.07146.

208. Xie, S.; Girshick, R.; Dollár, P.; Tu, Z.; He, K. Aggregated residual transformations for deep neural networks. In Proceedings of the IEEE Conference on Computer Vision and Pattern Recognition (CVPR), Honolulu, HI, USA, 21-26 July 2017; pp. 1492-1500.

209. Gao, S.; Cheng, M.M.; Zhao, K.; Zhang, X.Y.; Yang, M.H.; Torr, P.H. Res2Net: A New Multi-scale Backbone Architecture. IEEE Trans. Pattern Anal. Mach. Intell. 2019, 43, 652-662. [CrossRef] [PubMed]

210. Szegedy, C.; Ioffe, S.; Vanhoucke, V.; Alemi, A. Inception-v4, inception-resnet and the impact of residual connections on learning. arXiv 2016, arXiv:1602.07261.

211. Huang, J.; Rathod, V.; Sun, C.; Zhu, M.; Korattikara, A.; Fathi, A.; Fischer, I.; Wojna, Z.; Song, Y.; Guadarrama, S.; et al. Speed/Accuracy Trade-Offs for Modern Convolutional Object Detectors. In Proceedings of the 2017 IEEE Conference on Computer Vision and Pattern Recognition (CVPR), Honolulu, HI, USA, 21-26 July 2017; pp. 3296-3297.

212. Madokoro, H.; Yamamoto, S.; Nishimura, Y.; Nix, S.; Woo, H.; Sato, K. Prototype Development of Small Mobile Robots for Mallard Navigation in Paddy Fields: Toward Realizing Remote Farming. Robotics 2021, 10, 63. [CrossRef]

213. Kingma, D.P.; Ba, J. Adam: A Method for Stochastic Optimization. arXiv 2014, arXiv:1412.6980v9.

214. Qian, N. On the momentum term in gradient descent learning algorithms. Neural Netw. 1999, 12, 145-151. [CrossRef]

215. Tieleman, T.; Hinton, G. Lecture 6.5-RMSProp: Divide the Gradient by a Running Average of its Recent Magnitude. COURSERA Neural Netw. Mach. Learn. 2012, 4, 26-31.

216. Gross, J.J.; John, O.P. Individual Differences in Two Emotion Regulation Processes: Implications for Affect, Relationships, and Well-Being. J. Personal. Soc. Psychol. 2003, 85, 348-362. [CrossRef] [PubMed]

217. Saari, M.; bin Baharudin, A.M.; Hyrynsalmi, S. Survey of prototyping solutions utilizing Raspberry Pi. In Proceedings of the 40th International Convention on Information and Communication Technology, Electronics and Microelectronics (MIPRO), Opatija, Croatia, 22-26 May 2017; pp. 991-994.

218. Yang, D.Q.B.; Gu, N. Indoor multiple human targets localization and tracking using thermopile sensor. Infrared Phys. Technol. 2019, 97, 349-359

219. Madokoro, H.; Kiguchi, O.; Nagayoshi, T.; Chiba, T.; Inoue, M.; Chiyonobu, S.; Nix, S.; Woo, H.; Sato, K. Development of Drone-Mounted Multiple Sensing System with Advanced Mobility for In Situ Atmospheric Measurement: A Case Study Focusing on $\mathrm{PM}_{2.5}$ Local Distribution. Sensors 2021, 21, 4881. [CrossRef] [PubMed]

220. Hochreiter, S. The Vanishing Gradient Problem During Learning Recurrent Neural Nets and Problem Solutions. Int. J. Uncertain. Fuzziness Knowl.-Based Syst. 1998, 6, 107-116. [CrossRef] 